Article

\title{
Hesitant Neutrosophic Linguistic Sets and Their Application in Multiple Attribute Decision Making
}

\author{
Yongfeng Pang and Wei Yang * \\ Department of Mathematics, School of Science, Xi'an University of Architecture and Technology, \\ Xi'an 710055, China; pangyongfeng75@163.com \\ * Correspondence: yangweipyf@163.com or yangwei_lxy@xauat.edu.cn; Tel.: +86-029-8220-2739
}

Received: 13 March 2018; Accepted: 5 April 2018; Published: 11 April 2018

\begin{abstract}
In this paper, the hesitant neutrosophic linguistic set is first defined by extending a hesitant fuzzy set to accommodate linguistic terms and neutrosophic fuzzy values. Some operational laws are defined for hesitant neutrosophic linguistic fuzzy information. Several distance measures have been defined including generalized hesitant neutrosophic linguistic distance, generalized hesitant neutrosophic linguistic Hausdorff distance, and generalized hesitant neutrosophic linguistic hybrid distance. Some hesitant neutrosophic fuzzy linguistic aggregation operators based on the Choquet integral have been defined. A new multiple attribute decision making method for hesitant neutrosophic fuzzy linguistic information has been developed based on TOPSIS. In order to illustrate the feasibility and practical advantages of the new algorithm, we use it to select a company to invest. The new method is then compared with other methods.
\end{abstract}

Keywords: neutrosophic set; linguistic argument; hesitant fuzzy set; Choquet integral; multiple attribute decision making

\section{Introduction}

With the development of society and the economy, decision problems have become increasingly complicated. Fuzziness and uncertainty exists extensively in the decision process. Many useful tools have been developed to model fuzzy and uncertain information, including fuzzy sets, intuitionistic fuzzy sets, interval-valued intuitionistic fuzzy sets, linguistic arguments, hesitant fuzzy sets, and neutrosophic sets. Many useful methods have been developed based on these tools. Smarandache [1] developed the neutrosophic set by generalizing a fuzzy set, an intuitionistic fuzzy set [2], and a hesitant fuzzy set. The neutrosophic set has received extensively attention [3-25] recently. The neutrosophic fuzzy set has been extended to accommodate interval values $[7,8]$, linguistic variables $[9,10]$, and trapezoidal fuzzy numbers [11]. Ye [9] proposed the concept of a single valued neutrosophic linguistic set as a generalization of the concepts of a linguistic variable and an intuitionistic linguistic set. Ye [11] combined trapezoidal fuzzy numbers with a single-valued neutrosophic set to propose a trapezoidal neutrosophic set and defined some trapezoidal neutrosophic fuzzy aggregation operators. The neutrosophic fuzzy set has been used in image segmentation [12], clustering analysis [13], supply chain management [14], etc. Guo and Sengür [12] utilized the neutrosophic set in image segmentation and proposed a novel algorithm based on neutrosophic similarity clustering. Karaaslan [13] defined the concept of a single-valued neutrosophic refined soft set by extending a single-valued neutrosophic refined set. Some aggregation operators have been developed [3,7,15-18]. Liu and Tang [3] proposed some power average aggregation operators for interval neutrosophic sets, including the interval neutrosophic power generalized weighted aggregation operator and interval neutrosophic power generalized ordered weighted aggregation operator. Zhao et al. [7] defined the generalized weighted aggregation operator for interval valued neutrosophic sets based on a traditional 
generalized weighted aggregation operator. Liu and Shi [15] defined the interval neutrosophic hesitant fuzzy set and developed some new interval neutrosophic hesitant fuzzy generalized hybrid aggregation operators. Liu et al. [16] proposed some generalized neutrosophic number hamacher weighted aggregation operators. Sun et al. [17] defined a ranking index to compare two interval neutrosophic numbers and defined an interval neutrosophic Choquet integral operator. Peng et al. [18] developed the multi-valued neutrosophic sets and defined the operations of multi-valued neutrosophic numbers based on Einstein operations. Some distances, cross entropies and correlation coefficient measures have been defined [19-26]. Ye [19] defined the Hamming and Euclidean distances between interval neutrosophic sets and proposed the similarity measures based on the relationship between similarity measures and distances. Ye presented single-valued neutrosophic cross entropy in [20] and presented the correlation coefficient of single-valued neutrosophic sets based on the extension of the intuitionistic fuzzy correlation coefficient in [21]. Zhang et al. [22] proposed a new correlation coefficient measure for interval neutrosophic sets. Şahin [23] defined the interval neutrosophic cross-entropy based on extension of fuzzy cross entropy and single-valued neutrosophic cross entropy. Tian et al. [26] proposed a fuzzy cross entropy approach to calculate the discrimination measure between alternatives and the absolute ideal solutions.

A hesitant fuzzy set is another extension of the fuzzy set, which was developed by Torra and Narukawa $[27,28]$. In the hesitant fuzzy set, the membership degree of an element to a set is represented by several possible values. Compared with other tools to model fuzzy and uncertain information, the hesitant fuzzy set is more flexible and accurate, especially for large-scale and complex decision problems. The hesitant fuzzy set has received a great deal of attention [29-34]. Due to the fuzzy nature of human thinking, decision-makers would like to evaluate with linguistic terms. Several linguistic decision making models have been developed [35-39], including the 2-tuple linguistic model [35], uncertain linguistic variables [36], the hesitant fuzzy linguistic terms set [37], and the linguistic hesitant intuitionistic fuzzy term set [39]. With the development of the economy and society, decision problems become more and more complex, and multiple decision-makers from different fields are invited to evaluate. Decision-makers (DMs) would like to use linguistic terms in the evaluation process, but they may express some hesitation, which can be modeled properly by the neutrosophic set. Since each DM has his/her own characteristics, he/she can give proper evaluation values if he/she is familiar with the attribute and can refuse to produce any evaluation values if he/she is unfamiliar with the attribute in order to assure accuracy and reasonability of decision results. DMs can use a different number of linguistic terms and a different number of neutrosophic fuzzy values in evaluating the attribute. Then, we can get hesitant neutrosophic linguistic information. Ye [40] developed hesitant interval neutrosophic linguistic set and proposed the hesitant interval neutrosophic linguistic weighted average operator and a hesitant interval neutrosophic linguistic weighted geometric operator. It is rather difficult to use interval linguistic values in evaluation, and distances between hesitant interval neutrosophic linguistic elements have not been studied. To our best knowledge, hesitant neutrosophic linguistic sets, which are very important in decision-making, especially for complex decision-making problems requiring fast evaluation, have not been studied. Motivated by the idea of the hesitant fuzzy set, the neutrosophic set, and linguistic terms, we present hesitant neutrosophic linguistic sets. Then, we define some operational laws and some distant measures between hesitant neutrosophic linguistic sets. A new multiple attribute decision-making method based on hesitant neutrosophic linguistic information has thus been developed.

The rest of the paper is organized as follows. In Section 2, we first briefly review some concepts on the hesitant fuzzy set, the linguistic variable, and the neutrosophic set. Then, we propose a definition of the hesitant neutrosophic linguistic set and provide some operation laws of hesitant neutrosophic linguistic elements. We also define the cosine value between two hesitant neutrosophic linguistic elements to compare them. In Section 3, we present some distance measures for hesitant neutrosophic linguistic elements. In Section 4, we develop some hesitant neutrosophic linguistic aggregation operators based on the Choquet integral. In Section 5, we present a new multiple attribute group 
decision-making method based on TOPSIS for hesitant neutrosophic fuzzy linguistic information. A numerical example is presented in Section 6, and some comparisons are also made. Conclusions are presented in Section 7.

\section{Preliminaries}

In this section, we give the definition of the hesitant neutrosophic linguistic set and present some operation laws. We also present the method to compare two hesitant neutrosophic linguistic elements by using their cosine values.

In the decision-making process, there are cases where a DM, in determining the membership of an element to a set, doubts several different values. Torra and Narukawa $[27,28]$ generalized a fuzzy set to make each membership include several values and developed the hesitant fuzzy set (HFS). An HFS is defined in terms of a function that returns a set of membership values of each element in the domain.

Definition 1. Let $X$ be a reference set. An HFS $\tilde{A}$ on $X$ is a function $m$ that returns a subset of values in $[0,1]$ when it is applied to $\mathrm{X}$ :

$$
\tilde{A}=\left\{<x, m_{\tilde{A}}(x)>\mid x \in X\right\}
$$

where $m_{\tilde{A}}(x)$ is a set of some different values in $[0,1]$, representing the possible membership degrees of element $x \in X$ to $\tilde{A} . m_{\tilde{A}}(x)$ is called a hesitant fuzzy element (HFE).

Let $S=\left\{s_{i} \mid i=1, \ldots, g\right\}$ be a finite and totally ordered discrete term set, where $s_{i}$ represents a possible value for a linguistic variable. For example, a set of nine terms [36] can be expressed as

$$
\begin{aligned}
S= & \left\{s_{1}=\text { extremely poor, } s_{2}=\text { very poor, } s_{3}=\text { poor, } s_{4}=\text { slightly poor, } s_{5}=\right.\text { fair, } \\
& \left.s_{6}=\text { slightly good, } s_{7}=\text { good, } s_{8}=\text { very good, } s_{9}=\text { extremely good }\right\}
\end{aligned}
$$

The above set satisfies the following properties:

(1) The set is ordered: $s_{i} \geq s_{j}$, if $i \geq j$.

(2) Max operator: $\max \left\{s_{i}, s_{j}\right\}=s_{i}$, if $i \geq j$.

(3) Min operator: $\min \left\{s_{i}, s_{j}\right\}=s_{i}$, if $i \leq j$.

(4) Negation operator: $\operatorname{neg}\left\{s_{i}\right\}=s_{g-i}$.

In order to preserve all the information, the discrete linguistic term sets $S$ can be extended to a continuous one: $\bar{S}=\left\{s_{\alpha} \mid s_{0} \leq s_{\alpha} \leq s_{g}\right\}, \alpha \in[0, g]$.

Definition 2. [1] Let X be a universeof discourse, with a generic element in X denoted by $x$. A neutrosophic set $A$ in $X$ is

$$
A=\left\{x\left(T_{A}(x), I_{A}(x), F_{A}(x)\right) \mid x \in X\right\},
$$

where $T_{A}$ is the truth-membership function, $I_{A}$ is the indeterminacy-membership function, $F_{A}$ is the real falsity-membership function. $T_{A}(x), I_{A}(x)$ and $F_{A}(x)$ are real standard or nonstandard subsets of $] 0^{-}, 1^{+}[$. There is no restriction on the sum of $T_{A}(x), I_{A}(x)$ and $F_{A}(x)$, we can obtain $0^{-} \leq T_{A}(x)+I_{A}(x)+$ $F_{A}(x) \leq 3^{+}$.

DMs would like to evaluate with linguistic terms since such terms reflect the fuzzy nature of human thinking. For complex decision-making problems, multiple experts from different fields are invited to evaluate alternatives with respect to multiple attributes. If the expert is familiar with the attribute, he/she can provide a proper evaluation value. If he/she is not familiar with the attribute, he/she can refuse to provide any evaluation value to assure the reasonability of the results. If two experts use the same truth-membership, indeterminacy-membership, or real falsity-membership, they are merged. We can then obtain the hesitant neutrosophic linguistic set as follows. 
Definition 3. Let $X=\left\{x_{1}, x_{2}, \ldots, x_{n}\right\}$ be a reference set. A hesitant neutrosophic linguistic set (HNLS) $A$ in $X$ is

$$
A=\left\{<x_{i}, h_{A}\left(x_{i}\right)>\mid x_{i} \in X, i=1,2, \ldots, n\right\}
$$

where $h_{A}\left(x_{i}\right)=\left\{\left(s_{\theta_{i}},\left(T\left(s_{\theta_{i}}\right), I\left(s_{\theta_{i}}\right), F\left(s_{\theta_{i}}\right)\right)\right)\right\}, T\left(s_{\theta_{i}}\right)$ is the set of truth-membership functions, $I\left(s_{\theta_{i}}\right)$ is the set of indeterminacy-membership functions, and $F\left(s_{\theta_{i}}\right)$ is the set of real falsity-membership functions. $\sup \left(T\left(s_{\theta_{i}}\right)\right)+\sup \left(I\left(s_{\theta_{i}}\right)\right)+\sup \left(F\left(s_{\theta_{i}}\right)\right) \leq 3$. For convenience, we call $h_{A}\left(x_{i}\right)$ the hesitant neutrosophic linguistic element (HNLE). We can use $a=\left(s_{\theta_{i}},\left(T\left(s_{\theta_{i}}\right), I\left(s_{\theta_{i}}\right)\right.\right.$, and $\left.\left.F\left(s_{\theta_{i}}\right)\right)\right)$ to represent a neutrosophic linguistic element (NLE).

Definition 4. Let $h, h_{1}$, and $h_{2}$ be HNLEs, and let $h=\left\{a_{k}\right\}, h_{1}=\left\{a_{i}^{(1)}\right\}, h_{2}=\left\{a_{j}^{(2)}\right\}$, $a_{k}=\left(s_{\theta_{k}}\left(T\left(s_{\theta_{k}}\right), I\left(s_{\theta_{k}}\right), F\left(s_{\theta_{k}}\right)\right)\right) \in h, a_{i}^{(1)}=\left(s_{\theta_{i}^{(1)}},\left(T\left(s_{\theta_{i}^{(1)}}\right), I\left(s_{\theta_{i}^{(1)}}\right), F\left(s_{\theta_{i}^{(1)}}\right)\right)\right) \in h_{1}$ and $a_{j}^{(2)}=$ $\left(s_{\theta_{j}^{(2)}},\left(T\left(s_{\theta_{j}^{(2)}}\right), I\left(s_{\theta_{j}^{(2)}}\right), F\left(s_{\theta_{j}^{(2)}}\right)\right)\right) \in h_{2}$ be NLEs. Let $\mu_{i}^{(1)} \in F\left(s_{\theta_{i}^{(1)}}\right), \mu_{j}^{(2)} \in F\left(s_{\theta_{j}^{(2)}}\right), v_{i}^{(1)} \in T\left(s_{\theta_{i}^{(1)}}\right), v_{j}^{(2)} \in$ $T\left(s_{\theta_{j}^{(2)}}\right), \tau_{i}^{(1)} \in I\left(s_{\theta_{i}^{(1)}}\right), \tau_{j}^{(2)} \in I\left(s_{\theta_{j}^{(2)}}\right) . \lambda>0$. The operational laws can be defined as follows.

(1) $h_{1} \oplus h_{2}=\bigcup_{a_{i}^{(1)} \in h_{1}, a_{j}^{(2)} \in h_{2}}\left\{\left(s_{\theta_{i}^{(1)}+\theta_{j}^{(2)}}, \bigcup\left\{\left(\mu_{i}^{(1)}+\mu_{j}^{(2)}-\mu_{i}^{(1)} \mu_{j}^{(2)}, v_{i}^{(1)} v_{j}^{(2)}, \tau_{i}^{(1)} \tau_{j}^{(2)}\right)\right\}\right)\right\}$

(2) $\quad h_{1} \otimes h_{2}=\bigcup_{a_{i}^{(1)} \in h_{1}, a_{j}^{(2)} \in h_{2}}\left\{\left(s_{\theta_{i}^{(1)} \theta_{j}^{(2)}}, \bigcup\left\{\left(\mu_{i}^{(1)} \mu_{j}^{(2)}, v_{i}^{(1)}+v_{j}^{(2)}-v_{i}^{(1)} v_{j}^{(2)}, \tau_{i}^{(1)}+\tau_{j}^{(2)}-\tau_{i}^{(1)} \tau_{j}^{(2)}\right)\right\}\right)\right\}$

(3) $\lambda h=\bigcup_{a_{k} \in h}\left\{\left(s_{\lambda \theta_{k}}, \bigcup\left\{\left(1-\left(1-\mu_{k}\right)^{\lambda},\left(v_{k}\right)^{\lambda},\left(\tau_{k}\right)^{\lambda}\right)\right\}\right)\right\}$

(4) $\left.h^{\lambda}=\bigcup_{a_{k} \in h}\left\{\left(s_{\theta_{k}^{\lambda}}, \bigcup\left\{\left(\mu_{k}\right)^{\lambda}, 1-\left(1-v_{k}\right)^{\lambda}, 1-\left(1-\tau_{k}\right)^{\lambda}\right)\right\}\right)\right\}$.

Theorem 1. Let $h, h_{1}, h_{2}$, and $h_{3}$ be HNLEs, $\lambda, \lambda_{1}$ and $\lambda_{2}>0$. Then,

(1) $h_{1} \oplus h_{2}=h_{2} \oplus h_{1}$

(2) $h_{1} \otimes h_{2}=h_{2} \otimes h_{1}$

(3) $\lambda\left(h_{1} \oplus h_{2}\right)=\lambda h_{1} \oplus \lambda h_{2}$

(4) $\left(\lambda_{1}+\lambda_{2}\right) h=\lambda_{1} h \oplus \lambda_{2} h$

(5) $\left(h_{1} \otimes h_{2}\right)^{\lambda}=h_{1}^{\lambda} \otimes h_{2}^{\lambda}$

(6) $h^{\lambda_{1}+\lambda_{2}}=h^{\lambda_{1}} \otimes h^{\lambda_{2}}$

(7) $\left(h_{1} \oplus h_{2}\right) \oplus h_{3}=h_{1} \oplus\left(h_{2} \oplus h_{3}\right)$

(8) $\left(h_{1} \otimes h_{2}\right) \otimes h_{3}=h_{1} \otimes\left(h_{2} \otimes h_{3}\right)$.

Different HNLEs may have different NLEs, and different NLEs may have different truthmemberships, indeterminacy-memberships, or falsity-memberships. In order to define the distances between two HNLEs more accurately, we need to extend the HNLEs until they have the same number of NLEs and until each NLE has the same number of truth-memberships, indeterminacy-memberships, and falsity-memberships. We can extend the HNLEs according to the risk attitudes of the decisionmakers. If decision-makers are risk-seeking, the largest NLE can be added; if decision-makers are risk-averse, the smallest NLE can be added; if decision-makers are risk-neutral, the average value of NLEs can be added.

Definition 5. Let $a_{i}$ and $a_{j}$ be NLEs. $a_{i}=\left(s_{\theta_{i}},\left(T\left(s_{\theta_{i}}\right), I\left(s_{\theta_{i}}\right), F\left(s_{\theta_{i}}\right)\right)\right), a_{j}=\left(s_{\theta_{j}},\left(T\left(s_{\theta_{j}}\right), I\left(s_{\theta_{j}}\right), F\left(s_{\theta_{j}}\right)\right)\right)$, $\mu_{i k} \in T\left(s_{\theta_{i}}\right), v_{i l} \in I\left(s_{\theta_{i}}\right), \tau_{i m} \in F\left(s_{\theta_{i}}\right), \mu_{j k} \in T\left(s_{\theta_{j}}\right), v_{j l} \in I\left(s_{\theta_{j}}\right), \tau_{j m} \in F\left(s_{\theta_{j}}\right)$. The cosine value between $a_{i}$ and $a_{j}$ can be defined as follows:

$$
\cos \left(a_{i}, a_{j}\right)=\frac{\theta_{i} \theta_{j} / g^{2}+\frac{1}{l_{t}} \sum_{k=1}^{l_{t}} \mu_{i \sigma(k)} \mu_{j \sigma(k)}+\frac{1}{l_{i}} \sum_{k=1}^{l_{i}} v_{i \sigma(k)} v_{j \sigma(k)}+\frac{1}{l_{f}} \sum_{k=1}^{l_{f}} \tau_{i \sigma(k)} \tau_{j \sigma(k)}}{4\left\|a_{i}\right\| \cdot\left\|a_{j}\right\|}
$$


where $\left\|a_{i}\right\|=\left(\frac{1}{4}\left(\theta_{i}^{2} / g^{2}+\frac{1}{l_{t}} \sum_{k=1}^{l_{t}} \mu_{i k}^{2}+\frac{1}{l_{i}} \sum_{k=1}^{l_{i}} v_{i k}^{2}+\frac{1}{l_{f}} \sum_{k=1}^{l_{f}} \tau_{i k}^{2}\right)\right)^{1 / 2},\left\|a_{j}\right\|=\left(\frac{1}{4}\left(\theta_{j}^{2} / g^{2}+\frac{1}{l_{t}} \sum_{k=1}^{l_{t}} \mu_{j k}^{2}\right.\right.$ $\left.\left.+\frac{1}{l_{i}} \sum_{k=1}^{l_{i}} v_{j k}^{2}+\frac{1}{l_{f}} \sum_{k=1}^{l_{f}} \tau_{j k}^{2}\right)\right)^{1 / 2}$, $g$ is the number of linguistic variables in the linguistic term set, $l_{t}$ is the number of truth-memberships in the NLE, $l_{i}$ is the number of indeterminacy-membership in the NLE, and $l_{f}$ is the number of falsity-membership in the NLE. $0<\cos \left(a_{i}, a_{j}\right) \leq 1$. If $a_{j}$ is the ideal solution $I=$ $\left(s_{g},\{1, \ldots, 1\},\{0, \ldots, 0\},\{0, \ldots, 0\}\right)$, then

$$
\cos \left(a_{i}, I\right)=\frac{\theta_{i} / g+\frac{1}{l_{t}} \sum_{k=1}^{l_{t}} \mu_{i \sigma(k)}}{\sqrt{2\left(\theta_{i}^{2} / g^{2}+\frac{1}{l_{t}} \sum_{k=1}^{l_{t}} \mu_{i k}^{2}+\frac{1}{l_{i}} \sum_{k=1}^{l_{i}} v_{i k}^{2}+\frac{1}{l_{f}} \sum_{k=1}^{l_{f}} \tau_{i k}^{2}\right)}}
$$

Definition 6. Let $a_{1}, a_{2}$ be two NLEs. If $\cos \left(a_{1}, I\right) \leq \cos \left(a_{2}, I\right)$, then $a_{1} \leq a_{2}$.

Definition 7. Let $h_{1}, h_{2}$ be HNLEs. $h_{1}=\left\{a_{i}^{(1)}\right\}=\left\{\left(s_{\theta_{i}^{(1)}},\left(T\left(s_{\theta_{i}^{(1)}}\right), I\left(s_{\theta_{i}^{(1)}}\right), F\left(s_{\theta_{i}^{(1)}}\right)\right)\right)\right\}, h_{2}=\left\{a_{i}^{(2)}\right\}=$ $\left\{\left(s_{\theta_{i}^{(2)}},\left(T\left(s_{\theta_{i}^{(2)}}\right), I\left(s_{\theta_{i}^{(2)}}\right), F\left(s_{\theta_{i}^{(1)}}\right)\right)\right)\right\}$. The cosine value between $h_{1}$ and $h_{2}$ can be defined as follows:

$$
\cos \left(h_{1}, h_{2}\right)=\frac{h_{1} \cdot h_{2}}{\left\|h_{1}\right\| \cdot\left\|h_{2}\right\|}
$$

where

$$
\begin{gathered}
h_{1} \cdot h_{2}=\frac{1}{4 l} \sum_{i=1}^{l}\left(\left(\theta_{\sigma(i)}^{(1)} \theta_{\sigma(i)}^{(2)}\right) / g^{2}+\frac{1}{l_{t}} \sum_{k=1}^{l_{t}}\left(\mu_{\sigma(i k)}^{(1)} \mu_{\sigma(i k)}^{(2)}\right)+\frac{1}{l_{i}} \sum_{k=1}^{l_{i}}\left(v_{\sigma(i k)}^{(1)} v_{\sigma(i k)}^{(2)}\right)^{2}+\frac{1}{l_{t}} \sum_{k=1}^{l_{t}}\left(\tau_{\sigma(i k)}^{(1)} \tau_{\sigma(i k)}^{(1)}\right)^{2}\right) \\
\left\|h_{1}\right\|=\left(\frac{1}{4 l} \sum_{i=1}^{l}\left(\left(\theta_{i}^{(1)}\right)^{2} / g^{2}+\frac{1}{l_{t}} \sum_{k=1}^{l_{t}}\left(\mu_{i k}^{(1)}\right)^{2}+\frac{1}{l_{i}} \sum_{k=1}^{l_{i}}\left(v_{i k}^{(1)}\right)^{2}+\frac{1}{l_{f}} \sum_{k=1}^{l_{f}}\left(\tau_{i k}^{(1)}\right)^{2}\right)\right)^{1 / 2} \\
\left\|h_{2}\right\|=\left(\frac{1}{4 l} \sum_{i=1}^{l}\left(\left(\theta_{i}^{(2)}\right)^{2} / g^{2}+\frac{1}{l_{t}} \sum_{k=1}^{l_{t}}\left(\mu_{i k}^{(2)}\right)^{2}+\frac{1}{l_{i}} \sum_{k=1}^{l_{i}}\left(v_{i k}^{(2)}\right)^{2}+\frac{1}{l_{f}} \sum_{k=1}^{l_{f}} *\left(\tau_{i k}^{(2)}\right)^{2}\right)\right)^{1 / 2} .
\end{gathered}
$$

$l$ is the number of NLEs in the HNLEs, $g$ is the number of linguistic variables in the linguistic term set, $l_{t}$ is the number of truth-memberships in an NLE, $l_{i}$ is the number of indeterminacy-membership in an $N L E, l_{f}$ is the number of falsity-membership in an NLE. $0<\cos \left(h_{1}, h_{2}\right) \leq 1$. When $h_{2}$ is the ideal solution $I^{\prime}=\left\{\left(s_{g},\{1, \ldots, 1\},\{0, \ldots, 0\},\{0, \ldots, 0\}\right)\right\}$, the bigger $\cos \left(h_{1}, I^{\prime}\right)$ between $h_{1}$ and $I^{\prime}$ is, the more consistent the direction between $h_{1}$ and $I^{\prime}$ is. Thus,

$$
\cos \left(h_{1}, I^{\prime}\right)=\frac{\frac{1}{l} \sum_{i=1}^{l}\left(\left(\theta_{\sigma(i)}^{(1)}\right) / g+\frac{1}{l_{t}} \sum_{k=1}^{l_{t}} \mu_{\sigma(i k)}^{(1)}\right)}{\sqrt{\frac{2}{l} \sum_{i=1}^{l}\left(\left(\theta_{i}^{(1)}\right)^{2} / g^{2}+\frac{1}{l_{t}} \sum_{k=1}^{l_{t}}\left(\mu_{i k}^{(1)}\right)^{2}+\frac{1}{l_{i}} \sum_{k=1}^{l_{i}}\left(v_{i k}^{(1)}\right)^{2}+\frac{1}{l_{t}} \sum_{k=1}^{l_{f}}\left(\tau_{i k}^{(1)}\right)^{2}\right)}} .
$$

Definition 8. Let $h_{1}, h_{2}$ be two HNLEs. If $\cos \left(h_{1}, I^{\prime}\right) \leq \cos \left(h_{2}, I^{\prime}\right)$, then $h_{1} \leq h_{2}$.

\section{Some Hesitant Neutrosophic Fuzzy Linguistic Distance Measures}

In this section, we propose some distance measures for the hesitant neutrosophic fuzzy linguistic values and we also propose some correlated distance measures by using the Choquet integral.

The distance measures are very important since they are the basis of many well-known methods including TOPSIS, VIKOR, and ELECTRE. In this section, we develop some distance measures for hesitant neutrosophic fuzzy linguistic information.

Definition 9. Let $h_{1}, h_{2}$ be HNLEs. The distance measure between $h_{1}$ and $h_{2}$ can be defined as $d\left(h_{1}, h_{2}\right)$, which satisfies the following properties:

(1) $0 \leq d\left(h_{1}, h_{2}\right) \leq 1$;

(2) $d\left(h_{1}, h_{2}\right)=0$ if and only if $h_{1}=h_{2}$;

(3) $d\left(h_{1}, h_{2}\right)=d\left(h_{2}, h_{1}\right)$. 
Definition 10. Let $h_{1}, h_{2}$ be HNLEs. Then the similarity measure between $h_{1}$ and $h_{2}$ is defined as $\rho\left(h_{1}, h_{2}\right)$, which satisfies the following properties:

(1) $0 \leq \rho\left(h_{1}, h_{2}\right) \leq 1$;

(2) $\rho\left(h_{1}, h_{2}\right)=1$ if and only if $h_{1}=h_{2}$;

(3) $\rho\left(h_{1}, h_{2}\right)=\rho\left(h_{2}, h_{1}\right)$.

Remark 1. If $d\left(h_{1}, h_{2}\right)$ is the distance between $h_{1}$ and $h_{2}$, then $\rho\left(h_{1}, h_{2}\right)=1-d\left(h_{1}, h_{2}\right)$ is the similarity measure between $h_{1}$ and $h_{2}$. Hence, we only focus on the distance measures, and the similarity measures can be determined easily.

Let $h_{1}, h_{2}$ be HNLEs. $h_{1}=\left\{a_{i}^{(1)}\right\}=\left\{\left(s_{\theta_{i}^{(1)}},\left(T\left(s_{\theta_{i}^{(1)}}\right), I\left(s_{\theta_{i}^{(1)}}\right), F\left(s_{\theta_{i}^{(1)}}\right)\right)\right)\right\}, T\left(s_{\theta_{i}^{(1)}}\right)=\left\{\mu_{i t_{i}}^{(1)}\right\}$, $I\left(s_{\theta_{i}^{(1)}}\right)=\left\{v_{i t_{i}}^{(1)}\right\}, F\left(s_{\theta_{i}^{(1)}}\right)=\left\{\tau_{i t_{i}}^{(1)}\right\}$ and $h_{2}=\left\{a_{i}^{(2)}\right\}=\left\{\left(s_{\theta_{i}^{(2)}},\left(T\left(s_{\theta_{i}^{(2)}}\right), I\left(s_{\theta_{i}^{(2)}}\right), F\left(s_{\theta_{i}^{(1)}}\right)\right)\right)\right\}, T\left(s_{\theta_{i}^{(2)}}\right)=$ $\left\{\mu_{i t_{i}}^{(2)}\right\}, I\left(s_{\theta_{i}^{(2)}}\right)=\left\{v_{i t_{i}}^{(2)}\right\}, F\left(s_{\theta_{i}^{(2)}}\right)=\left\{\tau_{i t_{i}}^{(2)}\right\} \cdot a_{\sigma(i)}^{(k)} \geq a_{\sigma(j)}^{(k)}$ for $i \geq j . l$ is the number of NLEs in HNLE, $g$ is the number of linguistic variables in the linguistic term set, $l_{t}$ is the number of truth-memberships in an NLE, $l_{i}$ is the number of indeterminacy-membership in an NLE, $l_{f}$ is the number of falsity-membership in an NLE. Based on the operation laws of HNLEs and the extension principle, we define some distance measures between HNLEs.

The hesitant neutrosophic linguistic Euclidean distance between $h_{1}$ and $h_{2}$ can be defined as follows:

$$
\begin{aligned}
d_{E}\left(h_{1}, h_{2}\right)= & \left(\frac { 1 } { 4 l } \sum _ { i = 1 } ^ { l } \left(\left(\theta_{\sigma(i)}^{(1)}-\theta_{\sigma(i)}^{(2)}\right)^{2} / g^{2}+\frac{1}{l_{t}} \sum_{k=1}^{l_{t}}\left(\mu_{\sigma(i k)}^{(1)}-\mu_{\sigma(i k)}^{(2)}\right)^{2}+\frac{1}{l_{i}} \sum_{k=1}^{l_{i}}\left(\nu_{\sigma(i k)}^{(1)}-\right.\right.\right. \\
& \left.\left.\left.v_{\sigma(i k)}^{(2)}\right)^{2}+\frac{1}{l_{t}} \sum_{k=1}^{l_{t}}\left(\tau_{\sigma(i k)}^{(1)}-\tau_{\sigma(i k)}^{(2)}\right)^{2}\right)\right)^{1 / 2} .
\end{aligned}
$$

The hesitant neutrosophic linguistic Hamming distance between $h_{1}$ and $h_{2}$ can be defined as follows:

$$
\begin{aligned}
d_{H}\left(h_{1}, h_{2}\right)= & \frac{1}{4 l} \sum_{i=1}^{l}\left(\left|\theta_{\sigma(i)}^{(1)}-\theta_{\sigma(i)}^{(2)}\right| / g+\frac{1}{l_{t}} \sum_{k=1}^{l_{t}}\left|\mu_{\sigma(i k)}^{(1)}-\mu_{\sigma(i k)}^{(2)}\right|+\frac{1}{l_{i}} \sum_{k=1}^{l_{i}} \mid v_{\sigma(i k)}^{(1)}-\right. \\
& \left.v_{\sigma(i k)}^{(2)}\left|+\frac{1}{l_{t}} \sum_{k=1}^{l_{t}}\right| \tau_{\sigma(i k)}^{(1)}-\tau_{\sigma(i k)}^{(2)} \mid\right) .
\end{aligned}
$$

The hesitant neutrosophic linguistic Hamming-Hausdorff distance can be defined as follows:

$$
\begin{aligned}
d_{H H}\left(h_{1}, h_{2}\right)= & \frac{1}{4} \max _{i, k}\left\{\left|\theta_{\sigma(i)}^{(1)}-\theta_{\sigma(i)}^{(2)}\right| / g+\left|\mu_{\sigma(i k)}^{(1)}-\mu_{\sigma(i k)}^{(2)}\right|+\left|v_{\sigma(i k)}^{(1)}-v_{\sigma(i k)}^{(2)}\right|+\right. \\
& \left.\left|\tau_{\sigma(i k)}^{(1)}-\tau_{\sigma(i k)}^{(2)}\right|\right\} .
\end{aligned}
$$

The hesitant neutrosophic linguistic Euclidean-Hausdorff distance can be defined as

$$
\begin{aligned}
d_{E H}\left(h_{1}, h_{2}\right)= & \left(\frac { 1 } { 4 } \operatorname { m a x } _ { i , k } \left\{\left(\theta_{\sigma(i)}^{(1)}-\theta_{\sigma(i)}^{(2)}\right)^{2} / g^{2}+\left|\mu_{\sigma(i k)}^{(1)}-\mu_{\sigma(i k)}^{(2)}\right|^{2}+\left|v_{\sigma(i k)}^{(1)}-v_{\sigma(i k)}^{(2)}\right|^{2}+\right.\right. \\
& \left.\left.\left|\tau_{\sigma(i k)}^{(1)}-\tau_{\sigma(i k)}^{(2)}\right|^{2}\right\}\right)^{1 / 2} .
\end{aligned}
$$

The hybrid hesitant neutrosophic linguistic Hamming distance can be defined as

$$
\begin{aligned}
d_{H y b H}\left(h_{1}, h_{2}\right)= & \frac{1}{2}\left(\frac { 1 } { 4 l } \sum _ { i = 1 } ^ { l } \left(\left|\theta_{\sigma(i)}^{(1)}-\theta_{\sigma(i)}^{(2)}\right| / g+\frac{1}{l_{t}} \sum_{k=1}^{l_{t}}\left|\mu_{\sigma(i k)}^{(1)}-\mu_{\sigma(i k)}^{(2)}\right|+\frac{1}{l_{i}} \sum_{k=1}^{l_{i}} \mid \nu_{\sigma(i k)}^{(1)}-\right.\right. \\
& \left.v_{\sigma(i k)}^{(2)}\left|+\frac{1}{l_{t}} \sum_{k=1}^{l_{t}}\right| \tau_{\sigma(i k)}^{(1)}-\tau_{\sigma(i k)}^{(2)} \mid\right)+\frac{1}{4} \max _{i, k}\left\{\left|\theta_{\sigma(i)}^{(1)}-\theta_{\sigma(i)}^{(2)}\right| / g+\mid \mu_{\sigma(i k)}^{(1)}-\right. \\
& \left.\left.\mu_{\sigma(i k)}^{(2)}|+| \nu_{\sigma(i k)}^{(1)}-v_{\sigma(i k)}^{(2)}|+| \tau_{\sigma(i k)}^{(1)}-\tau_{\sigma(i k)}^{(2)} \mid\right\}\right) .
\end{aligned}
$$

The hybrid hesitant neutrosophic linguistic Euclidean distance can be defined as 


$$
\begin{aligned}
d_{\text {HybE }}\left(h_{1}, h_{2}\right)= & \frac{1}{2}\left(\left(\frac { 1 } { 4 l } \sum _ { i = 1 } ^ { l } \left(\left(\theta_{\sigma(i)}^{(1)}-\theta_{\sigma(i)}^{(2)}\right)^{2} / g^{2}+\frac{1}{l_{t}} \sum_{k=1}^{l_{t}}\left(\mu_{\sigma(i k)}^{(1)}-\mu_{\sigma(i k)}^{(2)}\right)^{2}+\frac{1}{l_{i}} \sum_{k=1}^{l_{i}}\left(v_{\sigma(i k)}^{(1)}-\right.\right.\right.\right. \\
& \left.\left.\left.v_{\sigma(i k)}^{(2)}\right)^{2}+\frac{1}{l_{t}} \sum_{k=1}^{l_{t}}\left(\tau_{\sigma(i k)}^{(1)}-\tau_{\sigma(i k)}^{(2)}\right)^{2}\right)\right)^{1 / 2}+\left(\frac { 1 } { 4 } \operatorname { m a x } _ { i , k } \left\{\left(\theta_{\sigma(i)}^{(1)}-\theta_{\sigma(i)}^{(2)}\right)^{2} / g^{2}+\mid \mu_{\sigma(i k)}^{(1)}-\right.\right. \\
& \left.\left.\left.\left.\mu_{\sigma(i k)}^{(2)}\right|^{2}+\left|v_{\sigma(i k)}^{(1)}-v_{\sigma(i k)}^{(2)}\right|^{2}+\left|\tau_{\sigma(i k)}^{(1)}-\tau_{\sigma(i k)}^{(2)}\right|^{2}\right\}\right)^{1 / 2}\right) .
\end{aligned}
$$

The generalized hesitant neutrosophic linguistic distance can be defined as

$$
\begin{aligned}
d_{G}\left(h_{1}, h_{2}\right)= & \left(\frac { 1 } { 4 l } \sum _ { i = 1 } ^ { l } \left(\left(\theta_{\sigma(i)}^{(1)}-\theta_{\sigma(i)}^{(2)}\right)^{\lambda} / g^{\lambda}+\frac{1}{l_{t}} \sum_{k=1}^{l_{t}}\left(\mu_{\sigma(i k)}^{(1)}-\mu_{\sigma(i k)}^{(2)}\right)^{\lambda}+\frac{1}{l_{i}} \sum_{k=1}^{l_{i}}\left(v_{\sigma(i k)}^{(1)}-\right.\right.\right. \\
& \left.\left.\left.v_{\sigma(i k)}^{(2)}\right)^{\lambda}+\frac{1}{l_{t}} \sum_{k=1}^{l_{t}}\left(\tau_{\sigma(i k)}^{(1)}-\tau_{\sigma(i k)}^{(2)}\right)^{\lambda}\right)\right)^{1 / \lambda} .
\end{aligned}
$$

If $\lambda=1$, the generalized hesitant neutrosophic linguistic distance becomes the hesitant neutrosophic linguistic Hamming distance. If $\lambda=2$, then the generalized hesitant neutrosophic linguistic distance becomes the hesitant neutrosophic linguistic Euclidean distance.

The generalized hesitant neutrosophic linguistic Hausdorff distance can be defined as

$$
\begin{aligned}
d_{G H}\left(h_{1}, h_{2}\right)= & \left(\frac { 1 } { 4 } \operatorname { m a x } _ { i , k } \left\{\left|\theta_{\sigma(i)}^{(1)}-\theta_{\sigma(i)}^{(2)}\right|^{\lambda} / g^{\lambda}+\left|\mu_{\sigma(i k)}^{(1)}-\mu_{\sigma(i k)}^{(2)}\right|^{\lambda}+\left|v_{\sigma(i k)}^{(1)}-v_{\sigma(i k)}^{(2)}\right|^{\lambda}+\right.\right. \\
& \left.\left.\left|\tau_{\sigma(i k)}^{(1)}-\tau_{\sigma(i k)}^{(2)}\right|^{\lambda}\right\}\right)^{1 / \lambda} .
\end{aligned}
$$

If $\lambda=1$, the generalized hesitant neutrosophic linguistic Hausdorff distance becomes the hesitant neutrosophic linguistic Hamming-Hausdorff distance. If $\lambda=2$, the generalized hesitant neutrosophic linguistic Hausdorff distance becomes the hesitant neutrosophic linguistic Euclidean-Hausdorff distance.

The generalized hesitant neutrosophic linguistic hybrid distance can be defined as

$$
\begin{aligned}
d_{G H y b}\left(h_{1}, h_{2}\right)= & \left(\frac { 1 } { 2 } \left(\frac { 1 } { 4 l } \sum _ { i = 1 } ^ { l } \left(\left(\theta_{\sigma(i)}^{(1)}-\theta_{\sigma(i)}^{(2)}\right)^{\lambda} / g^{\lambda}+\frac{1}{l_{t}} \sum_{k=1}^{l_{t}}\left(\mu_{\sigma(i k)}^{(1)}-\mu_{\sigma(i k)}^{(2)}\right)^{\lambda}+\frac{1}{l_{i}} \sum_{k=1}^{l_{i}}\left(v_{\sigma(i k)}^{(1)}\right.\right.\right.\right. \\
& \left.\left.-v_{\sigma(i k)}^{(2)}\right)^{\lambda}+\frac{1}{l_{t}} \sum_{k=1}^{l_{t}}\left(\tau_{\sigma(i k)}^{(1)}-\tau_{\sigma(i k)}^{(2)}\right)^{\lambda}\right)+\frac{1}{4} \max _{i, k}\left\{\left|\theta_{\sigma(i)}^{(1)}-\theta_{\sigma(i)}^{(2)}\right|^{\lambda} / g^{\lambda}+\mid \mu_{\sigma(i k)}^{(1)}\right. \\
& \left.\left.\left.-\left.\mu_{\sigma(i k)}^{(2)}\right|^{\lambda}+\left|v_{\sigma(i k)}^{(1)}-v_{\sigma(i k)}^{(2)}\right|^{\lambda}+\left|\tau_{\sigma(i k)}^{(1)}-\tau_{\sigma(i k)}^{(2)}\right|^{\lambda}\right\}\right)\right)^{1 / \lambda} .
\end{aligned}
$$

Definition 11. [41]. A fuzzy measure $\mu$ on the set $X$ is a set function $\mu: P(X) \rightarrow[0,1]$, satisfying the following axioms:

(1) $\mu(\varnothing)=0, \mu(X)=1$;

(2) $B \subseteq C$ implies $\mu(B) \leq \mu(C)$, for all $B, C \subseteq X$;

(3) $\mu(B \cup C)=\mu(B)+\mu(C)+\rho \mu(B) \mu(C)$ for all $B, C \subseteq X$ and $B \cap C=\varnothing$, where $\rho \in(-1,+\infty)$.

Here, $\varnothing$ is an empty set.

Let $X=\left\{x_{1}, x_{2}, \ldots, x_{n}\right\}$ be a finite set. Sugeno [42] provided the following equation to determine the fuzzy measure on $X$, avoiding the computational complexity.

$$
m(X)= \begin{cases}\frac{1}{\rho}\left(\Pi_{i=1}^{n}\left(1+\rho m\left(x_{i}\right)\right)-1\right), & \rho \neq 0, \\ \sum_{i=1}^{n} m\left(x_{i}\right), & \rho=0 .\end{cases}
$$

The value $\rho$ can be uniquely determined from $m(X)=1$ by the following equation:

$$
\rho=\Pi_{i=1}^{n}\left(1+\rho m\left(x_{i}\right)\right)-1 .
$$

Let $X=\left\{x_{1}, x_{2}, \ldots, x_{n}\right\}$ be a reference set, let $m$ be the fuzzy measure [41] on $X$, and let $H_{1}=\left\{h_{1}^{(1)}, h_{2}^{(1)}, \ldots, h_{n}^{(1)}\right\}$ and $H_{2}=\left\{h_{1}^{(2)}, h_{2}^{(2)}, \ldots, h_{n}^{(2)}\right\}$ be two collections of the HNLEs on $X$, 
where $h_{j}^{(k)}=\left\{a_{j}^{l(k)}\right\}(j=1,2, \ldots, n, k=1,2)$. We then develop some weighted distances between HNLEs based on the Choquet integral.

The generalized hesitant neutrosophic linguistic correlation averaging distance between $H_{1}$ and $\mathrm{H}_{2}$ can be defined as follows:

$$
\begin{aligned}
d_{G C}\left(H_{1}, H_{2}\right)= & \left(\sum _ { j = 1 } ^ { n } \frac { ( m ( A _ { \sigma ( j ) } ) - m ( A _ { \sigma ( j - 1 ) } ) ) } { 4 l } \sum _ { i = 1 } ^ { l } \left(\left(\theta_{\sigma(j i)}^{(1)}-\theta_{\sigma(j i)}^{(2)}\right)^{\lambda} / g^{\lambda}+\frac{1}{l_{t}} \sum_{k=1}^{l_{t}}\left(\mu_{\sigma(j i k)}^{(1)}-\right.\right.\right. \\
& \left.\left.\left.\mu_{\sigma(j i k)}^{(2)}\right)^{\lambda}+\frac{1}{l_{u}} \sum_{k=1}^{l_{u}}\left(v_{\sigma(j i k)}^{(1)}-v_{\sigma(j i k)}^{(2)}\right)^{\lambda}+\frac{1}{l_{v}} \sum_{k=1}^{l_{v}}\left(\tau_{\sigma(j i k)}^{(1)}-\tau_{\sigma(j i k)}^{(2)}\right)^{\lambda}\right)\right)^{1 / \lambda} .
\end{aligned}
$$

If $\lambda=1$, the generalized hesitant neutrosophic linguistic correlation averaging distance becomes the hesitant neutrosophic linguistic correlation averaging Hamming distance as follows:

$$
\begin{aligned}
d_{C H}\left(H_{1}, H_{2}\right)= & \sum_{j=1}^{n} \frac{\left(m\left(A_{\sigma(j)}\right)-m\left(A_{\sigma(j-1)}\right)\right)}{4 l} \sum_{i=1}^{l}\left(\left|\theta_{\sigma(j i)}^{(1)}-\theta_{\sigma(j i)}^{(2)}\right| / g+\frac{1}{l_{t}} \sum_{k=1}^{l_{t}} \mid \mu_{\sigma(j i k)}^{(1)}-\right. \\
& \left.\mu_{\sigma(j i k)}^{(2)}\left|+\frac{1}{l_{u}} \sum_{k=1}^{l_{u}}\right| v_{\sigma(j i k)}^{(1)}-v_{\sigma(j i k)}^{(2)}\left|+\frac{1}{l_{v}} \sum_{k=1}^{l_{v}}\right| \tau_{\sigma(j i k)}^{(1)}-\tau_{\sigma(j i k)}^{(2)} \mid\right) .
\end{aligned}
$$

If $\lambda=2$, the generalized hesitant neutrosophic linguistic correlation averaging distance becomes the hesitant neutrosophic linguistic correlation averaging Euclidean distance as follows:

$$
\begin{aligned}
d_{C E}\left(H_{1}, H_{2}\right)= & \left(\sum _ { j = 1 } ^ { n } \frac { ( m ( A _ { \sigma ( j ) } ) - m ( A _ { \sigma ( j - 1 ) } ) ) } { 4 l } \sum _ { i = 1 } ^ { l } \left(\left(\theta_{\sigma(j i)}^{(1)}-\theta_{\sigma(j i)}^{(2)}\right)^{2} / g^{2}+\frac{1}{l_{t}} \sum_{k=1}^{l_{t}}\left(\mu_{\sigma(j i k)}^{(1)}-\right.\right.\right. \\
& \left.\left.\left.\mu_{\sigma(j i k)}^{(2)}\right)^{2}+\frac{1}{l_{u}} \sum_{k=1}^{l_{u}^{u}}\left(v_{\sigma(j i k)}^{(1)}-v_{\sigma(j i k)}^{(2)}\right)^{2}+\frac{1}{l_{v}} \sum_{k=1}^{l_{v}}\left(\tau_{\sigma(j i k)}^{(1)}-\tau_{\sigma(j i k)}^{(2)}\right)^{2}\right)\right)^{1 / 2} .
\end{aligned}
$$

The generalized hesitant neutrosophic linguistic correlation averaging Housdorff distance is defined as follows:

$$
\begin{aligned}
d_{G C H}\left(H_{1}, H_{2}\right)= & \left(\sum _ { j = 1 } ^ { n } \frac { ( m ( A _ { \sigma ( j ) } ) - m ( A _ { \sigma ( j - 1 ) } ) ) } { 4 } \operatorname { m a x } _ { i , k } \left\{\left|\theta_{\sigma(j i)}^{(1)}-\theta_{\sigma(j i)}^{(2)}\right|^{\lambda} / g^{\lambda}+\left|\mu_{\sigma(j i k)}^{(1)}-\mu_{\sigma(j i k)}^{(2)}\right|^{\lambda}\right.\right. \\
& \left.\left.+\left|v_{\sigma(j i k)}^{(1)}-v_{\sigma(j i k)}^{(2)}\right|^{\lambda}+\left|\tau_{\sigma(j i k)}^{(1)}-\tau_{\sigma(j i k)}^{(2)}\right|^{\lambda}\right\}\right)^{1 / \lambda} .
\end{aligned}
$$

If $\lambda=1$, the generalized hesitant neutrosophic linguistic correlation averaging Housdorff distance becomes the hesitant neutrosophic linguistic correlation averaging Hamming-Housdorff distance

$$
\begin{aligned}
d_{C H H}\left(H_{1}, H_{2}\right)= & \left(\sum _ { j = 1 } ^ { n } \frac { ( m ( A _ { \sigma ( j ) } ) - m ( A _ { \sigma ( j - 1 ) } ) ) } { 4 } \operatorname { m a x } _ { i , k } \left\{\left|\theta_{\sigma(j i)}^{(1)}-\theta_{\sigma(j i)}^{(2)}\right| / g+\left|\mu_{\sigma(j i k)}^{(1)}-\mu_{\sigma(j i k)}^{(2)}\right|\right.\right. \\
& \left.\left.+\left|v_{\sigma(j i k)}^{(1)}-v_{\sigma(j i k)}^{(2)}\right|+\left|\tau_{\sigma(j i k)}^{(1)}-\tau_{\sigma(j i k)}^{(2)}\right|\right\}\right) .
\end{aligned}
$$

If $\lambda=2$, the generalized hesitant neutrosophic linguistic correlation averaging Housdorff distance becomes the hesitant neutrosophic linguistic correlation averaging Euclidean-Housdorff distance:

$$
\begin{aligned}
d_{C E H}\left(H_{1}, H_{2}\right)= & \left(\sum _ { j = 1 } ^ { n } \frac { ( m ( A _ { \sigma ( j ) } ) - m ( A _ { \sigma ( j - 1 ) } ) ) } { 4 } \operatorname { m a x } _ { i , k } \left\{\left|\theta_{\sigma(j i)}^{(1)}-\theta_{\sigma(j i)}^{(2)}\right|^{2} / g^{2}+\left|\mu_{\sigma(j i k)}^{(1)}-\mu_{\sigma(j i k)}^{(2)}\right|^{2}\right.\right. \\
& \left.\left.+\left|v_{\sigma(j i k)}^{(1)}-v_{\sigma(j i k)}^{(2)}\right|^{2}+\left|\tau_{\sigma(j i k)}^{(1)}-\tau_{\sigma(j i k)}^{(2)}\right|^{2}\right\}\right)^{1 / 2} .
\end{aligned}
$$

The generalized hesitant neutrosophic linguistic hybrid correlation averaging distance between $H_{1}$ and $H_{2}$ can be defined as follows:

$$
\begin{aligned}
d_{G H C}\left(H_{1}, H_{2}\right)= & \left(\frac { 1 } { 2 } \left(\sum _ { j = 1 } ^ { n } \frac { 1 } { 4 } ( m ( A _ { \sigma ( j ) } ) - m ( A _ { \sigma ( j - 1 ) } ) ) \left(\frac { 1 } { l } \sum _ { i = 1 } ^ { l } \left(\left(\theta_{\sigma(j i)}^{(1)}-\theta_{\sigma(j i)}^{(2)}\right)^{\lambda} / g^{\lambda}+\right.\right.\right.\right. \\
& \frac{1}{l_{t}} \sum_{k=1}^{l_{t}}\left(\mu_{\sigma(j i k)}^{(1)}-\mu_{\sigma(j i k)}^{(2)}\right)^{\lambda}+\frac{1}{l_{u}} \sum_{k=1}^{l_{u}}\left(v_{\sigma(j i k)}^{(1)}-v_{\sigma(j i k)}^{(2)}\right)^{\lambda}+\frac{1}{l_{v}} \sum_{k=1}^{l_{v}}\left(\tau_{\sigma(j i k)}^{(1)}-\right. \\
& \left.\left.\tau_{\sigma(j i k)}^{(2)}\right)^{\lambda}\right)+\max _{i, k}\left\{\left|\theta_{\sigma(j i)}^{(1)}-\theta_{\sigma(j i)}^{(2)}\right|^{\lambda} / g^{\lambda}+\left|\mu_{\sigma(j i k)}^{(1)}-\mu_{\sigma(j i k)}^{(2)}\right|^{\lambda}\right. \\
& \left.\left.\left.+\left|v_{\sigma(j i k)}^{(1)}-v_{\sigma(j i k)}^{(2)}\right|^{\lambda}+\left|\tau_{\sigma(j i k)}^{(1)}-\tau_{\sigma(j i k)}^{(2)}\right|^{\lambda}\right\}\right)\right)^{1 / \lambda} .
\end{aligned}
$$


If $\lambda=1$, the generalized hesitant neutrosophic linguistic hybrid correlation averaging distance becomes the hesitant neutrosophic linguistic hybrid correlation Hamming distance:

$$
\begin{aligned}
d_{C H y b}\left(H_{1}, H_{2}\right)= & \frac{1}{2}\left(\sum _ { j = 1 } ^ { n } \frac { 1 } { 4 } ( m ( A _ { \sigma ( j ) } ) - m ( A _ { \sigma ( j - 1 ) } ) ) \left(\frac { 1 } { l } \sum _ { i = 1 } ^ { l } \left(\left(\theta_{\sigma(j i)}^{(1)}-\theta_{\sigma(j i)}^{(2)}\right) / g+\right.\right.\right. \\
& \frac{1}{l_{t}} \sum_{k=1}^{l_{t}}\left(\mu_{\sigma(j i k)}^{(1)}-\mu_{\sigma(j i k)}^{(2)}\right)+\frac{1}{l_{u}} \sum_{k=1}^{l_{u}}\left(v_{\sigma(j i k)}^{(1)}-v_{\sigma(j i k)}^{(2)}\right)+\frac{1}{l_{v}} \sum_{k=1}^{l_{v}}\left(\tau_{\sigma(j i k)}^{(1)}-\right. \\
& \left.\left.\tau_{\sigma(j i k)}^{(2)}\right)\right)+\max _{i, k}\left\{\left|\theta_{\sigma(j i)}^{(1)}-\theta_{\sigma(j i)}^{(2)}\right| / g+\left|\mu_{\sigma(j i k)}^{(1)}-\mu_{\sigma(j i k)}^{(2)}\right|\right. \\
& \left.\left.+\left|v_{\sigma(j i k)}^{(1)}-v_{\sigma(j i k)}^{(2)}\right|+\left|\tau_{\sigma(j i k)}^{(1)}-\tau_{\sigma(j i k)}^{(2)}\right|\right\}\right) .
\end{aligned}
$$

If $\lambda=2$, the generalized hesitant neutrosophic linguistic hybrid correlation averaging distance becomes the hesitant neutrosophic linguistic hybrid correlation Euclidean distance:

$$
\begin{aligned}
d_{C H E}\left(H_{1}, H_{2}\right)= & \left(\frac { 1 } { 2 } \left(\sum _ { j = 1 } ^ { n } \frac { 1 } { 4 } ( m ( A _ { \sigma ( j ) } ) - m ( A _ { \sigma ( j - 1 ) } ) ) \left(\frac { 1 } { l } \sum _ { i = 1 } ^ { l } \left(\left(\theta_{\sigma(j i)}^{(1)}-\theta_{\sigma(j i)}^{(2)}\right)^{2} / g^{2}+\right.\right.\right.\right. \\
& \frac{1}{l_{t}} \sum_{k=1}^{l_{t}}\left(\mu_{\sigma(j i k)}^{(1)}-\mu_{\sigma(j i k)}^{(2)}\right)^{2}+\frac{1}{l_{u}} \sum_{k=1}^{l_{u}}\left(v_{\sigma(j i k)}^{(1)}-v_{\sigma(j i k)}^{(2)}\right)^{2}+\frac{1}{l_{v}} \sum_{k=1}^{l_{v}}\left(\tau_{\sigma(j i k)}^{(1)}-\right. \\
& \left.\left.\tau_{\sigma(j i k)}^{(2)}\right)^{2}\right)+\max _{i, k}\left\{\left|\theta_{\sigma(j i)}^{(1)}-\theta_{\sigma(j i)}^{(2)}\right|^{2} / g^{2}+\left|\mu_{\sigma(j i k)}^{(1)}-\mu_{\sigma(j i k)}^{(2)}\right|^{2}\right. \\
& \left.\left.\left.+\left|v_{\sigma(j i k)}^{(1)}-v_{\sigma(j i k)}^{(2)}\right|^{2}+\left|\tau_{\sigma(j i k)}^{(1)}-\tau_{\sigma(j i k)}^{(2)}\right|^{2}\right\}\right)\right)^{1 / 2} .
\end{aligned}
$$

\section{Some Hesitant Neutrosophic Fuzzy Linguistic Aggregation Operators Based on the Choquet Integral}

In this section, we present some hesitant neutrosophic fuzzy linguistic aggregation operators considering correlation by using the Choquet integral.

Definition 12. Let $X=\left\{x_{1}, x_{2}, \ldots, x_{n}\right\}$ be a reference set, and let $m$ be the fuzzy measure [41] on $X$. Let $h_{j}=\left\{a_{j}^{k}\right\}(j=1,2, \ldots, n)$ be a collection of the HNLEs, then the hesitant neutrosophic fuzzy linguistic correlation averaging (HNLCA) operator can be defined as follows:

$$
\operatorname{HNLCA}\left(h_{1}, h_{2}, \ldots, h_{n}\right)=\sum_{j=1}^{n}\left(m\left(A_{\sigma(j)}\right)-m\left(A_{\sigma(j-1)}\right)\right) h_{\sigma(j)}
$$

where $(\sigma(1), \sigma(2), \ldots, \sigma(n))$ is a permutation of $(1,2, \ldots, n)$ such that $h_{\sigma(1)} \geq h_{\sigma(2)} \geq \ldots \geq h_{\sigma(n)}$. $A_{\sigma(i)}=\left\{x_{\sigma(1)}, x_{\sigma(2)}, \ldots, x_{\sigma(i)}\right\}, A_{\sigma(0)}=\varnothing$, and $\varnothing$ is an empty set.

The aggregated value of the HNLCA operator is also an HNLE.

$$
\begin{aligned}
& \operatorname{HNLCA}\left(h_{1}, h_{2}, \ldots, h_{n}\right)=\sum_{j=1}^{n}\left(m\left(A_{\sigma(j)}\right)-m\left(A_{\sigma(j-1)}\right)\right) h_{\sigma(j)} \\
& =\bigcup_{a_{j}^{k} \in h_{j}}\left\{\left(s_{\sum_{j=1}^{n}\left(m\left(A_{\sigma(j)}\right)-m\left(A_{\sigma(j-1)}\right)\right) \theta_{\sigma(j)}^{k}}, \bigcup\left\{\left(1-\prod_{j=1}^{n}\left(1-\mu_{\sigma(j)}^{k}\right)^{m\left(A_{\sigma(j)}\right)-m\left(A_{\sigma(j-1)}\right)}\right.\right. \text {, }\right.\right. \\
& \left.\left.\left.\left.\prod_{j=1}^{n}\left(v_{\sigma(j)}^{k}\right)^{m\left(A_{\sigma(j)}\right)-m\left(A_{\sigma(j-1)}\right)}, \prod_{j=1}^{n}\left(\tau_{\sigma(j)}^{k}\right)^{m\left(A_{\sigma(j)}\right)-m\left(A_{\sigma(j-1)}\right)}\right)\right\}\right)\right\} .
\end{aligned}
$$

Definition 13. Let $X=\left\{x_{1}, x_{2}, \ldots, x_{n}\right\}$ be a reference set, and let $m$ be the fuzzy measure on $X$. Let $h_{j}=\left\{a_{j}^{k}\right\}$ $(j=1,2, \ldots, n)$ be a collection of the HNLEs. Thus, the hesitant neutrosophic fuzzy linguistic correlation geometric averaging (HNLCGA) operator can be defined as follows:

$$
\operatorname{HNLCGA}\left(h_{1}, h_{2}, \ldots, h_{n}\right)=\prod_{j=1}^{n}\left(h_{\sigma(j)}\right)^{\left(m\left(A_{\sigma(j)}\right)-m\left(A_{\sigma(j-1)}\right)\right)},
$$

where $(\sigma(1), \sigma(2), \ldots, \sigma(n))$ is a permutation of $(1,2, \ldots, n)$ such that $h_{\sigma(1)} \geq h_{\sigma(2)} \geq \ldots \geq h_{\sigma(n)}$. $A_{\sigma(i)}=$ $\left\{x_{\sigma(1)}, x_{\sigma(2)}, \ldots, x_{\sigma(i)}\right\}, A_{\sigma(0)}=\varnothing$, and $\varnothing$ is an empty set.

The aggregated value of the HNLCGA operator is also an HNLE. 


$$
\begin{aligned}
& \operatorname{HNLCGA}\left(h_{1}, h_{2}, \ldots, h_{n}\right)=\prod_{j=1}^{n}\left(h_{\sigma(j)}\right)^{\left(m\left(A_{\sigma(j)}\right)-m\left(A_{\sigma(j-1)}\right)\right)} \\
= & \bigcup_{a_{j}^{k} \in h_{j}}\left\{\left(s \prod_{j=1}^{n}\left(\theta_{\sigma(j)}^{k}\right)^{\left(m\left(A_{\sigma(j)}\right)-m\left(A_{\sigma(j-1)}\right)\right)}, \bigcup\left\{\left(\prod_{j=1}^{n}\left(\mu_{\sigma(j)}^{k}\right)^{m\left(A_{\sigma(j)}\right)-m\left(A_{\sigma(j-1)}\right),}\right.\right.\right.\right. \\
& \left.\left.\left.\left.1-\prod_{j=1}^{n}\left(1-v_{\sigma(j)}^{k}\right)^{m\left(A_{\sigma(j)}\right)-m\left(A_{\sigma(j-1)}\right)}, 1-\prod_{j=1}^{n}\left(1-\tau_{\sigma(j)}^{k}\right)^{m\left(A_{\sigma(j)}\right)-m\left(A_{\sigma(j-1)}\right)}\right)\right\}\right)\right\} .
\end{aligned}
$$

We further develop the generalized hesitant neutrosophic fuzzy linguistic correlation averaging (GHNLCA) operator as follows.

Definition 14. Let $X=\left\{x_{1}, x_{2}, \ldots, x_{n}\right\}$ be a reference set, and let $m$ be the fuzzy measure on $X$. If $h_{j}=\left\{a_{j}^{k}\right\}$ $(j=1,2, \ldots, n)$ is a collection of the HNLEs, then the GHNLCA operator can be defined as follows:

$$
\operatorname{GHNLCA}\left(h_{1}, h_{2}, \ldots, h_{n}\right)=\left(\sum_{j=1}^{n}\left(m\left(A_{\sigma(j)}\right)-m\left(A_{\sigma(j-1)}\right)\right)\left(h_{\sigma(j)}\right)^{\lambda}\right)^{1 / \lambda},
$$

where $(\sigma(1), \sigma(2), \ldots, \sigma(n))$ is a permutation of $(1,2, \ldots, n)$ such that $h_{\sigma(1)} \geq h_{\sigma(2)} \geq \ldots \geq h_{\sigma(n)}$. $A_{\sigma(i)}=$ $\left\{x_{\sigma(1)}, x_{\sigma(2)}, \ldots, x_{\sigma(i)}\right\}, A_{\sigma(0)}=\varnothing, \varnothing$ is an empty set, and $\lambda>0$.

The aggregated value of the GHNLCA operator is also an HNLE.

$$
\begin{aligned}
& \operatorname{GHNLCA}\left(h_{1}, h_{2}, \ldots, h_{n}\right)=\left(\sum_{j=1}^{n} m\left(A_{\sigma(j)}\right)-m\left(A_{\sigma(j-1)}\right)\left(h_{\sigma(j)}\right)^{\lambda}\right)^{1 / \lambda}
\end{aligned}
$$

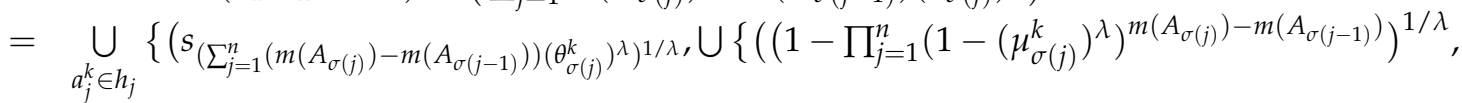

$$
\begin{aligned}
& 1-\left(1-\prod_{j=1}^{n}\left(1-\left(1-v_{\sigma(j)}^{k}\right)^{\lambda}\right)^{m\left(A_{\sigma(j)}\right)-m\left(A_{\sigma(j-1)}\right)}\right)^{1 / \lambda}, 1-\left(1-\prod_{j=1}^{n}(1-(1-\right. \\
& \left.\left.\left.\left.\left.\left.\left.\tau_{\sigma(j)}^{k}\right)^{\lambda}\right)^{m\left(A_{\sigma(j)}\right)-m\left(A_{\sigma(j-1)}\right)}\right)^{1 / \lambda}\right)\right\}\right)\right\} \text {. }
\end{aligned}
$$

Theorem 2. (Commutativity). If $\left(h_{1}^{\prime}, h_{2}^{\prime}, \ldots, h_{n}^{\prime}\right)$ is any permutation of $\left(h_{1}, h_{2}, \ldots, h_{n}\right)$, then

$$
\operatorname{GHNLCA}\left(h_{1}^{\prime}, h_{2}^{\prime}, \ldots, h_{n}^{\prime}\right)=\operatorname{GHNLCA}\left(h_{1}, h_{2}, \ldots, h_{n}\right) \text {. }
$$

\section{Proof of Theorem 2.}

$$
\begin{aligned}
& \operatorname{GHNLCA}\left(h_{1}, h_{2}, \ldots, h_{n}\right)=\left(\sum_{j=1}^{n} m\left(A_{\sigma(j)}\right)-m\left(A_{\sigma(j-1)}\right)\left(h_{\sigma(j)}\right)^{\lambda}\right)^{1 / \lambda}
\end{aligned}
$$

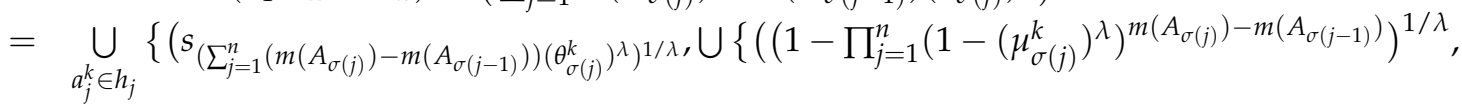

$$
\begin{aligned}
& 1-\left(1-\prod_{j=1}^{n}\left(1-\left(1-v_{\sigma(j)}^{k}\right)^{\lambda}\right)^{m\left(A_{\sigma(j)}\right)-m\left(A_{\sigma(j-1)}\right)}\right)^{1 / \lambda}, 1-\left(1-\prod_{j=1}^{n}(1-(1-\right. \\
& \left.\left.\left.\left.\left.\left.\left.\tau_{\sigma(j)}^{k}\right)^{\lambda}\right)^{m\left(A_{\sigma(j)}\right)-m\left(A_{\sigma(j-1)}\right)}\right)^{1 / \lambda}\right)\right\}\right)\right\}
\end{aligned}
$$

and

$$
\begin{aligned}
& \operatorname{GHNLCA}\left(h_{1}^{\prime}, h_{2}^{\prime}, \ldots, h_{n}^{\prime}\right)=\left(\sum_{j=1}^{n} m\left(A_{\sigma(j)}^{\prime}\right)-m\left(A_{\sigma(j-1)}^{\prime}\right)\left(h_{\sigma(j)}^{\prime}\right)^{\lambda}\right)^{1 / \lambda}
\end{aligned}
$$

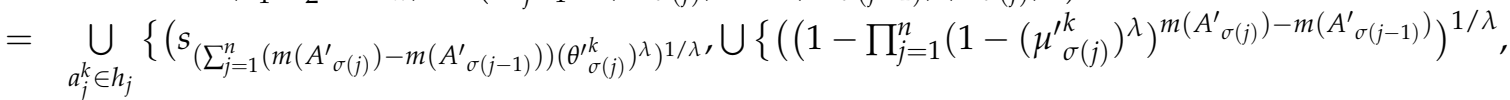

$$
\begin{aligned}
& 1-\left(1-\prod_{j=1}^{n}\left(1-\left(1-v_{\sigma(j)}^{\prime k}\right)^{\lambda}\right)^{m\left(A_{\sigma(j)}^{\prime}\right)-m\left(A_{\sigma(j-1)}^{\prime}\right)}\right)^{1 / \lambda}, 1-\left(1-\prod_{j=1}^{n}(1-(1-\right. \\
& \left.\left.\left.\left.\left.\left.\left.\tau_{\sigma(j)}^{\prime k}\right)^{\lambda}\right)^{m\left(A_{\sigma(j)}^{\prime}\right)-m\left(A_{\sigma(j-1)}^{\prime}\right)}\right)^{1 / \lambda}\right)\right\}\right)\right\} \text {. }
\end{aligned}
$$

If $\left(h_{1}^{\prime}, h_{2}^{\prime}, \ldots, h_{n}^{\prime}\right)$ is any permutation of $\left(h_{1}, h_{2}, \ldots, h_{n}\right)$, then we have $h_{\sigma(j)}=h_{\sigma(j)}^{\prime}$ and $m\left(A_{\sigma(j)}\right)=m\left(A_{\sigma(j)}^{\prime}\right)$.

$$
\operatorname{GHNLCA}\left(h_{1}^{\prime}, h_{2}^{\prime}, \ldots, h_{n}^{\prime}\right)=\operatorname{GHNLCA}\left(h_{1}, h_{2}, \ldots, h_{n}\right) .
$$


Theorem 3. (Idempotency). If $h_{j}=h, j=1,2, \ldots, n, h_{j}=\left\{a_{j}^{k}\right\}, a_{j}^{k}=\left(s_{\theta_{j}^{(k)}},\left(T\left(s_{\theta_{j}^{(k)}}\right), I\left(s_{\theta_{j}^{(k)}}\right), F\left(s_{\theta_{j}^{(k)}}\right)\right)\right)$, $T\left(s_{\theta_{j}^{(k)}}\right)=\left\{\mu_{j}^{(k)}\right\}, I\left(s_{\theta_{j}^{(k)}}\right)=\left\{v_{j}^{(k)}\right\}, F\left(s_{\theta_{j}^{(k)}}\right)=\left\{\tau_{j}^{(k)}\right\}$, then

$$
\operatorname{GHNLCA}\left(h_{1}, h_{2}, \ldots, h_{n}\right)=h \text {. }
$$

\section{Proof of Theorem 3.}

$$
\begin{aligned}
& \operatorname{GHNLCA}\left(h_{1}, h_{2}, \ldots, h_{n}\right)=\left(\sum_{j=1}^{n} m\left(A_{\sigma(j)}\right)-m\left(A_{\sigma(j-1)}\right)\left(h_{\sigma(j)}\right)^{\lambda}\right)^{1 / \lambda}
\end{aligned}
$$

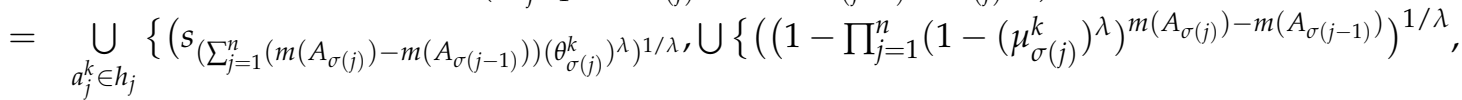

$$
\begin{aligned}
& 1-\left(1-\prod_{j=1}^{n}\left(1-\left(1-v_{\sigma(j)}^{k}\right)^{\lambda}\right)^{m\left(A_{\sigma(j)}\right)-m\left(A_{\sigma(j-1)}\right)}\right)^{1 / \lambda}, 1-\left(1-\prod_{j=1}^{n}(1-(1-\right. \\
& \left.\left.\left.\left.\left.\left.\left.\tau_{\sigma(j)}^{k}\right)^{\lambda}\right)^{m\left(A_{\sigma(j)}\right)-m\left(A_{\sigma(j-1)}\right)}\right)^{1 / \lambda}\right)\right\}\right)\right\} \text {. }
\end{aligned}
$$

If $h_{j}=h, j=1,2, \ldots, n$, then $a_{j}^{k}=a^{k}=\left(s_{\theta^{k}},\left(T\left(s_{\theta^{k}}\right), I\left(s_{\theta^{k}}\right), F\left(s_{\theta^{k}}\right)\right)\right), T\left(s_{\theta^{k}}\right)=\left\{\mu^{(k)}\right\}$, $I\left(s_{\theta^{k}}\right)=\left\{v^{(k)}\right\}, F\left(s_{\theta^{k}}\right)=\left\{\tau^{(k)}\right\}$. Thus,

$$
\begin{aligned}
& \left.S_{\left(\sum_{j=1}^{n}\left(m\left(A_{\sigma(j)}\right)-m\left(A_{\sigma(j-1)}\right)\right)\left(\theta^{k}\right)^{\lambda}\right)^{1 / \lambda}}=S_{\left(\left(\theta^{k}\right)^{\lambda}\right.} \sum_{j=1}^{n}\left(m\left(A_{\sigma(j)}\right)-m\left(A_{\sigma(j-1)}\right)\right)\right)^{1 / \lambda}=S_{\left(\left(\theta^{k}\right)^{\lambda}\right)^{1 / \lambda}}=s_{\theta^{k}} \\
& \left(1-\prod_{j=1}^{n}\left(1-\left(\mu_{\sigma(j)}^{k}\right)^{\lambda}\right)^{m\left(A_{\sigma(j)}\right)-m\left(A_{\sigma(j-1)}\right)}\right)^{1 / \lambda}=\left(1-\prod_{j=1}^{n}\left(1-\left(\mu^{(k)}\right)^{\lambda}\right)^{m\left(A_{\sigma(j)}\right)-m\left(A_{\sigma(j-1)}\right)}\right)^{1 / \lambda} \\
& =\left(1-\left(1-\left(\mu^{(k)}\right)^{\lambda}\right)^{\sum_{j=1}^{n}\left(m\left(A_{\sigma(j)}\right)-m\left(A_{\sigma(j-1)}\right)\right)}\right)^{1 / \lambda}=\left(1-\left(1-\left(\mu^{(k)}\right)^{\lambda}\right)^{\sum_{j=1}^{n}\left(m\left(A_{\sigma(j)}\right)-m\left(A_{\sigma(j-1)}\right)\right)}\right)^{1 / \lambda} \\
& =\left(1-\left(1-\left(\mu^{(k)}\right)^{\lambda}\right)\right)^{1 / \lambda}=\mu^{(k)} \text {. } \\
& 1-\left(1-\prod_{j=1}^{n}\left(1-\left(1-v_{\sigma(j)}^{(k)}\right)^{\lambda}\right)^{m\left(A_{\sigma(j)}\right)-m\left(A_{\sigma(j-1)}\right)}\right)^{1 / \lambda} \\
& =1-\left(1-\prod_{j=1}^{n}\left(1-\left(1-v^{(k)}\right)^{\lambda}\right)^{m\left(A_{\sigma(j)}\right)-m\left(A_{\sigma(j-1)}\right)}\right)^{1 / \lambda} \\
& =1-\left(1-\left(1-\left(1-v^{(k)}\right)^{\lambda}\right)^{\sum_{j=1}^{n}\left(m\left(A_{\sigma(j)}\right)-m\left(A_{\sigma(j-1)}\right)\right)}\right)^{1 / \lambda} \\
& =1-\left(1-\left(1-\left(1-v^{(k)}\right)^{\lambda}\right)\right)^{1 / \lambda}=1-\left(\left(1-v^{(k)}\right)^{\lambda}\right)^{1 / \lambda}=v^{(k)} \text {. } \\
& 1-\left(1-\prod_{j=1}^{n}\left(1-\left(1-\tau_{\sigma(j)}^{(k)}\right)^{\lambda}\right)^{m\left(A_{\sigma(j)}\right)-m\left(A_{\sigma(j-1)}\right)}\right)^{1 / \lambda} \\
& =1-\left(1-\prod_{j=1}^{n}\left(1-\left(1-\tau^{(k)}\right)^{\lambda}\right)^{m\left(A_{\sigma(j)}\right)-m\left(A_{\sigma(j-1)}\right)}\right)^{1 / \lambda} \\
& =1-\left(1-\left(1-\left(1-\tau^{(k)}\right)^{\lambda}\right)^{\sum_{j=1}^{n}\left(m\left(A_{\sigma(j)}\right)-m\left(A_{\sigma(j-1)}\right)\right)}\right)^{1 / \lambda} \\
& =1-\left(1-\left(1-\left(1-\tau^{(k)}\right)^{\lambda}\right)\right)^{1 / \lambda}=1-\left(\left(1-\tau^{(k)}\right)^{\lambda}\right)^{1 / \lambda}=\tau^{(k)} .
\end{aligned}
$$

Hence, GHNLCA $\left(h_{1}, h_{2}, \ldots, h_{n}\right)=h$.

Definition 15. Let $X=\left\{x_{1}, x_{2}, \ldots, x_{n}\right\}$ be a reference set, and let $m$ be the fuzzy measure on $X$. If $h_{j}=\left\{a_{j}^{k}\right\}$ $(j=1,2, \ldots, n)$ is a collection of the HNLEs, then the QHNLCA operator can be defined as follows:

$$
\operatorname{QHNLCA}\left(h_{1}, h_{2}, \ldots, h_{n}\right)=g^{-1}\left(\sum_{j=1}^{n}\left(m\left(A_{\sigma(j)}\right)-m\left(A_{\sigma(j-1)}\right)\right) g\left(h_{\sigma(j)}\right)\right)
$$

where $(\sigma(1), \sigma(2), \ldots, \sigma(n))$ is a permutation of $(1,2, \ldots, n)$ such that $h_{\sigma(1)} \geq h_{\sigma(2)} \geq \ldots \geq h_{\sigma(n)}$. $A_{\sigma(i)}=\left\{x_{\sigma(1)}, x_{\sigma(2)}, \ldots, x_{\sigma(i)}\right\}, A_{\sigma(0)}=\varnothing, \varnothing$ is an empty set, and $g(x)$ is a strictly monotonic continuous function.

\section{The Hesitant Neutrosophic Fuzzy Linguistic Decision-Making Method Based on TOPSIS}

For a multiple attribute decision-making problem with $p$ different periods $t_{k}(k=1,2, \ldots, p)$. Let $\left\{A_{1}, A_{2}, \ldots, A_{m}\right\}$ be the set of alternatives, and let $\left\{C_{1}, C_{2}, \ldots, C_{n}\right\}$ be the set of attributes. $h_{i j}^{k}=\left\{\left(s_{\theta_{i j}^{k}}\left(T\left(s_{\theta_{i j}^{k}}\right), I\left(s_{\theta_{i j}^{k}}\right), F\left(s_{\theta_{i j}^{k}}\right)\right)\right)\right\}$ is the hesitant neutrosophic linguistic evaluation value given 
by DMs in evaluating alternative $A_{i}$ with respect to the attribute $C_{j}$ in period $k$, where $s_{\theta_{i j}^{k}} \in S$, $\mu_{i j}^{k t} \in T\left(s_{\theta_{i j}^{k}}\right), v_{i j}^{k l} \in I\left(s_{\theta_{i j}^{k}}\right), \tau_{i j}^{k m} \in F\left(s_{\theta_{i j}^{k}}\right)$, and $0 \leq \sup \mu_{i j}^{k t}+\sup v_{i j}^{k l}+\sup \tau_{i j}^{k m} \leq 3$. The decision matrix is formed as $D^{k}=\left(h_{i j}^{k}\right)_{m \times n}(k=1,2, \ldots, p)$.

\section{Algorithm}

Step 1. The DMs evaluate alternatives $A_{i}(i=1,2, \ldots, m)$ with respect to attributes $C_{j}(j=$ $1,2, \ldots, n)$ in period $k(k=1,2, \ldots, p)$ with hesitant neutrosophic fuzzy linguistic elements and decision matrices are formed $H^{k}=\left(h_{i j}^{k}\right)_{m \times n}$.

$$
H^{k}=\left(\begin{array}{lrrr}
h_{11}^{k} & h_{12}^{k} & \ldots & h_{1 n}^{k} \\
h_{21}^{k} & h_{22}^{k} & \ldots & h_{2 n}^{k} \\
\vdots & \vdots & \ddots & \vdots \\
h_{m 1}^{k} & h_{m 2}^{k} & \ldots & h_{m n}^{k}
\end{array}\right) .
$$

Step 2. Confirm the fuzzy density of each period and determine $\rho$ by using the equation $\rho=$ $\left.\Pi_{i=1}^{n}\left(1+\rho m\left(t_{i}\right)\right)-1\right)$.

Step 3. Calculate the evaluation values $h_{i j}^{1}, h_{i j}^{2}, \ldots, h_{i j}^{p}$ in different periods into collective $h_{i j}$ by using the GHNLCA operator as follows:

$$
\begin{aligned}
h_{i j}= & \operatorname{GHNLCA}\left(h_{i j}^{1}, h_{i j}^{2}, \ldots, h_{i j}^{p}\right) \\
= & \bigcup_{a_{i j}^{k} \in h_{i j}^{k}}\left\{\left(s _ { ( \sum _ { k = 1 } ^ { p } ( m ( A _ { \sigma ( k ) } ) - m ( A _ { \sigma ( k - 1 ) } ) ) ( \theta _ { i j } ^ { ( k ) } ) ^ { \lambda } ) ^ { 1 / \lambda } } \cup \left\{\left(\left(1-\prod_{k=1}^{p}\left(1-\left(\mu_{i j}^{k t}\right)^{\lambda}\right)^{m\left(A_{\sigma(k)}\right)-m\left(A_{\sigma(k-1)}\right)}\right)^{1 / \lambda},\right.\right.\right.\right. \\
& 1-\left(1-\prod_{k=1}^{p}\left(1-\left(1-v_{i j}^{k l}\right)^{\lambda}\right)^{m\left(A_{\sigma(k)}\right)-m\left(A_{\sigma(k-1)}\right)}\right)^{1 / \lambda}, 1-\left(1-\prod_{k=1}^{p}(1-(1-\right. \\
& \left.\left.\left.\left.\left.\left.\left.\tau_{i j}^{k m}\right)^{\lambda}\right)^{m\left(A_{\sigma(k)}\right)-m\left(A_{\sigma(k-1)}\right)}\right)^{1 / \lambda}\right)\right\}\right)\right\}
\end{aligned}
$$

where $A_{\sigma(k)}=\left\{t_{1}, t_{2}, \ldots, t_{k}\right\}, A_{0}=\phi$. Thus, the decision matrix is formed as $H=\left(h_{i j}\right)_{m \times n}, h_{i j}=\left\{\left(s_{\theta_{i j}}\right.\right.$, $\left.\left.\left(T\left(s_{\theta_{i j}}\right), I\left(s_{\theta_{i j}}\right), F\left(s_{\theta_{i j}}\right)\right)\right)\right\}$.

Step 4. Extend the collective decision matrix according to risk attitudes of DMs until all HNLEs have the same number of NLEs and until each NLE has the same number of truth-memberships, indeterminacy-memberships, and falsity-memberships. The extended decision matrix is formed as $H^{\prime}=\left(h_{i j}^{\prime}\right)_{m \times n}$.

Step 5. Determine the hesitant neutrosophic linguistic positive ideal solution (HNLPIS), denoted as $h^{+}$, and the hesitant neutrosophic linguistic negative ideal solution (HNLNIS), denoted as $h^{-}$, as follows:

$$
\begin{aligned}
& h^{+}=\left(h_{1}^{+}, h_{2}^{+}, \ldots, h_{n}^{+}\right)=\left(\max _{i} h_{i 1}^{\prime}, \max _{i} h_{i 2}^{\prime}, \ldots, \max _{i} h_{i n}^{\prime}\right), \\
& h^{-}=\left(h_{1}^{-}, h_{2}^{-}, \ldots, h_{n}^{-}\right)=\left(\min _{i} h_{i 1}^{\prime}, \min _{i} h_{i 2}^{\prime}, \ldots, \min _{i} h_{i n}^{\prime}\right),
\end{aligned}
$$

where $h_{j}^{+}=\left\{\left(s_{\theta_{j}^{t+}},\left(T\left(s_{\theta_{j}^{t+}}\right), I\left(s_{\theta_{j}^{t+}}\right), F\left(s_{\theta_{j}^{t+}}\right)\right)\right)\right\}, T\left(s_{\theta_{j}^{t+}}\right)=\left\{\mu_{i j k}^{t+}\right\}, I\left(s_{\theta_{j}^{t+}}\right)=\left\{v_{i j k}^{t+}\right\}, F\left(s_{\theta_{j}^{t+}}\right)=\left\{\tau_{i j k}^{t+}\right\}$, $h_{j}^{-}=\left\{\left(s_{\theta_{j}^{t-}},\left(T\left(s_{\theta_{j}^{t-}}\right), I\left(s_{\theta_{j}^{t-}}\right), F\left(s_{\theta_{j}^{t-}}\right)\right)\right)\right\}, T\left(s_{\theta_{j}^{t-}}\right)=\left\{\mu_{i j k}^{t-}\right\}, I\left(s_{\theta_{j}^{t-}}\right)=\left\{v_{i j k}^{t-}\right\}, F\left(s_{\theta_{j}^{t-}}\right)=\left\{\tau_{i j k}^{t-}\right\}$.

Step 6. Confirm the fuzzy density $m\left(C_{i}\right)$ of each attribute. Determine $\rho$ by using Equation (14) and calculate fuzzy measures of attribute sets.

Step 7. Based on the generalized hesitant neutrosophic linguistic correlation weighted averaging distance, calculate the distances of each alternative's collective evaluation values to the HNLPIS $h^{+}$ and HNLNIS $h^{-}$, respectively. 


$$
\begin{aligned}
d_{G C}^{i+}\left(h_{i}, h^{+}\right)= & \left(\sum _ { j = 1 } ^ { n } ( m ( B _ { \sigma ( j ) } ) - m ( B _ { \sigma ( j - 1 ) } ) ) \frac { 1 } { 4 l } \sum _ { i = 1 } ^ { l } \left(\frac { 1 } { l _ { \theta } } \sum _ { t = 1 } ^ { l _ { \theta } } \left(\left|\theta_{\sigma(j i)}^{t}-\theta_{\sigma(j i)}^{t+}\right|^{\lambda} / g^{\lambda}+\right.\right.\right. \\
& \frac{1}{l_{t}} \sum_{k=1}^{l_{t}}\left|\mu_{\sigma(j i k)}^{t}-\mu_{\sigma(j i k)}^{t+}\right|^{\lambda}+\frac{1}{l_{u}} \sum_{k=1}^{l_{u}}\left|v_{\sigma(j i k)}^{t}-v_{\sigma(j i k)}^{t+}\right|^{\lambda}+\frac{1}{l_{v}} \sum_{k=1}^{l_{v}} \mid \tau_{\sigma(j i k)}^{t}- \\
& \left.\left.\left.\left.\tau_{\sigma(j i k)}^{t+}\right|^{\lambda}\right)\right)\right)^{1 / \lambda} \\
d_{G C}^{i-}\left(h_{i}, h^{-}\right)= & \left(\sum _ { j = 1 } ^ { n } ( m ( B _ { \sigma ( j ) } ) - m ( B _ { \sigma ( j - 1 ) } ) ) \left(\frac { 1 } { 4 l } \sum _ { i = 1 } ^ { l } \frac { 1 } { l _ { \theta } } \sum _ { t = 1 } ^ { l _ { \theta } } \left(\left|\theta_{\sigma(j i)}^{t}-\theta_{\sigma(j i)}^{t-}\right|^{\lambda} / g^{\lambda}+\right.\right.\right. \\
& \frac{1}{l_{t}} \sum_{k=1}^{l_{t}}\left|\mu_{\sigma(j i k)}^{t}-\mu_{\sigma(j i k)}^{t-}\right|^{\lambda}+\frac{1}{l_{u}} \sum_{k=1}^{l_{u}}\left|v_{\sigma(j i k)}^{t}-v_{\sigma(j i k)}^{t-}\right|^{\lambda}+\frac{1}{l_{v}} \sum_{k=1}^{l_{v}} \mid \tau_{\sigma(j i k)}^{t}- \\
& \left.\left.\left.\left.\tau_{\sigma(j i k)}^{t-}\right|^{\lambda}\right)\right)\right)^{1 / \lambda}
\end{aligned}
$$

where $d_{G C}^{1+}\left(h_{\sigma(i 1)}, h_{\sigma(1)}^{+}\right) \geq d_{G C}^{2+}\left(h_{\sigma(i 2)}, h_{\sigma(2)}^{+}\right) \geq \ldots \geq d_{G C}^{n+}\left(h_{\sigma(i n)}, h_{\sigma(n)}^{+}\right), d_{G C}^{1-}\left(h_{\sigma(i 1)}, h_{\sigma(1)}^{-}\right) \geq$ $d_{G C}^{2-}\left(h_{\sigma(i 2)}, h_{\sigma(2)}^{-}\right) \geq \ldots \geq d_{G C}^{n-}\left(h_{\sigma(i n)}, h_{\sigma(n)}^{-}\right), B_{\sigma(j)}=\left\{C_{\sigma(1)}, C_{\sigma(2)}, \ldots, C_{\sigma(j)}\right\}$, and $B_{\sigma(0)}=\phi, C_{\sigma(j)}$ is the attribute corresponding to the $j$ th largest $d_{G C}^{i+}\left(h_{i j}, h_{j}^{+}\right)$in $d_{G C}^{i+}$ or the $j$ th largest $d_{G C}^{i-}\left(h_{i j}, h_{j}^{-}\right)$in $d_{G C}^{i-}$.

Step 8. Calculate closeness coefficients $C C_{i}(i=1,2, \ldots, m)$ for alternatives $A_{i}(i=1,2, \ldots, m)$ as follows:

$$
C C_{i}=\frac{d_{G C}^{i-}\left(h_{i}, h^{-}\right)}{d_{G C}^{i+}\left(h_{i}, h^{-}\right)+d_{G C}^{i-}\left(h_{i}, h^{+}\right)}, i=1,2, \ldots, m .
$$

Step 9. Rank alternatives according to the ranking of closeness coefficients and select the optimal alternative.

\section{Numerical Example}

\subsection{Practical Example}

A company wants to invest a sum of money in an industry (adapted from [43]). The experts mainly consider the following attributes: $C_{1}$-the price of the product; $C_{2}$-the quality of the product; $C_{3}$-the risk; $C_{4}$-the delivery time. After pre-evaluation, there are five alternatives left for further evaluation. Alternatives are evaluated with respect to attributes using hesitant neutrosophic linguistic information. They consider information in three periods $\left(t_{i}, i=1,2, \ldots, 3\right)$. The new method is used to rank alternatives.

Step 1. The hesitant neutrosophic linguistic matrices $D^{k}(k=1,2,3)$ are given by experts in evaluating alternatives with respect to attributes in different periods as in Tables 1-3.

Step 2. Determine the fuzzy density of different $t_{i}$ and $\lambda$. Assume $m\left(t_{1}\right)=0.3, m\left(t_{2}\right)=0.4$, and $m\left(t_{3}\right)=0.5$. By using Equation (14), we can get $\rho=-0.3793$. Then we can get $m\left(t_{1}, t_{2}\right)=$ $0.6545, m\left(t_{1}, t_{3}\right)=0.7431, m\left(t_{2}, t_{3}\right)=0.8241$, and $m\left(t_{1}, t_{2}, t_{3}\right)=1.0$.

Step 3. Aggregate the collective evaluation values by using the GHNLCA operator. We can take different $\lambda$ values. Here, we consider the HNLCA operator by taking $\lambda=1$ in the GHNLCA operator. The results are shown in Table 4 .

Step 4. Assume decision-makers are risk-averse. Thus, the smallest linguistic evaluation value, the smallest truth-membership, the largest indeterminacy-membership, and the largest falsitymembership can be added until all the HNLEs have the same number of NLEs and until each NLE has the same number of truth-memberships, indeterminacy-memberships, and falsity-memberships. The results are shown in Table 5.

Step 5. We take the HNLPIS and the HNLNIS as follows:

HNLPIS: $h^{+}=\left\{\left\{\left(s_{9},(\{1,1\},\{0,0\},\{0,0\})\right),\left(s_{9},(\{1,1\},\{0,0\},\{0,0\})\right)\right\},\left\{\left(s_{9},(\{1,1\},\{0,0\},\{0,0\})\right)\right.\right.$, $\left.\left(s_{9},(\{1,1\},\{0,0\},\{0,0\})\right)\right\},\left\{\left(s_{9},(\{1,1\},\{0,0\},\{0,0\})\right),\left(s_{9},(\{1,1\},\{0,0\},\{0,0\})\right)\right\},\left\{\left(s_{9},(\{1,1\},\{0,0\}\right.\right.$, $\left.\left.\{0,0\})),\left(s_{9},(\{1,1\},\{0,0\},\{0,0\})\right)\right\}\right\}$.

HNLNIS: $h^{-}=\left\{\left\{\left(s_{1},\left(\{0,0\},\{1,1\},\{1,1\},\left(s_{1},(\{0,0\},\{1,1\},\{1,1\})\right)\right\},\left\{\left(s_{1},(\{0,0\},\{1,1\},\{1,1\}\right.\right.\right.\right.\right.$, $\left.\left(s_{1},(\{0,0\},\{1,1\},\{1,1\})\right)\right\},\left\{\left(s_{1},\left(\{0,0\},\{1,1\},\{1,1\},\left(s_{1},(\{0,0\},\{1,1\},\{1,1\})\right)\right\},\left\{\left(s_{1},(\{0,0\},\{1,1\},\{1,1\}\right.\right.\right.\right.$, $\left.\left.\left(s_{1},(\{0,0\},\{1,1\},\{1,1\})\right)\right\}\right\}$. 
Step 6. Assume $m\left(C_{1}\right)=0.25, m\left(C_{2}\right)=0.35, m\left(C_{3}\right)=0.30$, and $m\left(C_{4}\right)=0.20$. We can get $\rho=-0.2330$ by using Equation (14). Then, we can determine the fuzzy measures of the attribute sets as follows:

$m\left(\left\{C_{1}\right\}\right)=0.25, m\left(\left\{C_{2}\right\}\right)=0.35, m\left(\left\{C_{3}\right\}\right)=0.30, m\left(\left\{C_{4}\right\}\right)=0.20, m\left(\left\{C_{1}, C_{2}\right\}\right)=0.5796$, $m\left(\left\{C_{1}, C_{3}\right\}\right)=0.5325, m\left(\left\{C_{1}, C_{4}\right\}\right)=0.4383, m\left(\left\{C_{2}, C_{3}\right\}\right)=0.6255, m\left(\left\{C_{2}, C_{4}\right\}\right)=0.5337, m\left(\left\{C_{3}, C_{4}\right\}\right)=$ $0.4860, m\left(\left\{C_{1}, C_{2}, C_{3}\right\}\right)=0.8391, m\left(\left\{C_{1}, C_{2}, C_{4}\right\}\right)=0.7526, m\left(\left\{C_{1}, C_{3}, C_{4}\right\}\right)=0.7077, m\left(\left\{C_{2}, C_{3}, C_{4}\right\}\right)=$ $0.7964, m\left(\left\{C_{1}, C_{2}, C_{3}, C_{4}\right\}\right)=1.0$.

Step 7. Calculate the correlated distances of each alternative's evaluation values to the HNLPIS and the HNLNIS by generalized hesitant neutrosophic linguistic correlation weighted averaging distance. Here, we can take different $\lambda$ in Equations (30) and (31). If $\lambda=2$, we can get

$d_{C E}^{1+}=0.5249, d_{C E}^{2+}=0.5344, d_{C E}^{3+}=0.5082, d_{C E}^{4+}=0.5190, d_{C E}^{5+}=0.5249, d_{C E}^{1-}=0.5107, d_{C E}^{2-}=$ $0.5231, d_{C E}^{3-}=0.5432, d_{C E}^{4-}=0.5624, d_{C E}^{5-}=0.5439$.

Step 8. Calculate closeness coefficients of alternatives as

$C C_{1}=0.4932, C C_{2}=0.4947, C C_{3}=0.5166, C C_{4}=0.5200, C C_{5}=0.5262$.

Step 9. Rank alternatives according to the ranking of $C C_{i}$ to get $A_{5}>A_{4}>A_{3}>A_{2}>A_{1}$ and the optimal alternative is $A_{5}$.

If another $\lambda$ value is used in Step 7 , we can get distances, coefficients, and alternative rankings as in Table 6.

Table 1. Decision matrix $D^{1}$.

\begin{tabular}{ccc}
\hline Alternative & $C_{\mathbf{1}}$ & $C_{\mathbf{2}}$ \\
\hline$A_{1}$ & $\left\{\left(s_{5},(\{0.3,0.4\},\{0.2\},\{0.3\})\right)\right\}$ & $\left\{\left(s_{4},(\{0.4\},\{0.3\},\{0.2\})\right)\right\}$ \\
$A_{2}$ & $\left\{\left(s_{6},(\{0.6\},\{0.1\},\{0.2\})\right),\left(s_{7},(\{0.3\},\{0.4\},\{0.3\})\right)\right\}$ & $\left\{\left(s_{5},(\{0.6\},\{0.1\},\{0.2\})\right)\right\}$ \\
$A_{3}$ & $\left\{\left(s_{4},(\{0.3\},\{0.2\},\{0.3\})\right)\right\}$ & $\left\{\left(s_{3},(\{0.5\},\{0.2\},\{0.3\})\right),\left(s_{4},(\{0.3\},\{0.2\},\{0.5\})\right)\right\}$ \\
$A_{4}$ & $\left\{\left(s_{4},(\{0.7\},\{0.0\},\{0.1\})\right),\left(s_{5},(\{0.6\},\{0.2\},\{0.2\})\right)\right\}$ & $\left\{\left(s_{7},(\{0.6\},\{0.1\},\{0.2\})\right)\right\}$ \\
$A_{5}$ & $\left\{\left(s_{3},(\{0.5\},\{0.3,0.4\},\{0.2\})\right)\right\}$ & $\left\{\left(s_{2},(\{0.3\},\{0.3,0.4\},\{0.5\})\right)\right\}$ \\
\hline & $C_{3}$ & $C_{4}$ \\
\hline$A_{1}$ & $\left\{\left(s_{2},(\{0.2,0.3\},\{0.3\},\{0.5,0.6\})\right)\right\}$ & $\left\{\left(s_{5},(\{0.5\},\{0.2\},\{0.4\})\right),\left(s_{7},(\{0.4\},\{0.2\},\{0.3\})\right)\right\}$ \\
$A_{2}$ & $\left\{\left(s_{3},(\{0.5\},\{0.3\},\{0.2\})\right)\right\}$ & $\left\{\left(s_{6},(\{0.7,0.8\},\{0.1\},\{0.1\})\right)\right\}$ \\
$A_{3}$ & $\left\{\left(s_{5},(\{0.6\},\{0.1\},\{0.3\})\right)\right\}$ & $\left\{\left(s_{2},(\{0.4\},\{0.2\},\{0.3\})\right)\right\}$ \\
$A_{4}$ & $\left\{\left(s_{2},(\{0.7\},\{0.2\},\{0.1,0.2\})\right)\right\}$ & $\left\{\left(s_{3},(\{0.5\},\{0.3\},\{0.2\})\right)\right\}$ \\
$A_{5}$ & $\left\{\left(s_{8},(\{0.4\},\{0.3\},\{0.4\})\right)\right\}$ & $\left\{\left(s_{4},(\{0.5\},\{0.4\},\{0.3\})\right),\left(s_{5},(\{0.3\},\{0.3\},\{0.5\})\right)\right\}$ \\
\hline
\end{tabular}

Table 2. Decision matrix $D^{2}$.

\begin{tabular}{ccc}
\hline Alternative & $C_{\mathbf{1}}$ & $C_{\mathbf{2}}$ \\
\hline$A_{1}$ & $\left\{\left(s_{6},(\{0.2\},\{0.4\},\{0.3\})\right),\left(s_{7},(\{0.5\},\{0.1\},\{0.2\})\right)\right\}$ & $\left\{\left(s_{5},(\{0.2\},\{0.2,0.4\},\{0.5\})\right)\right\}$ \\
$A_{2}$ & $\left\{\left(s_{4},(\{0.4\},\{0.1\},\{0.3\})\right)\right\}$ & $\left\{\left(s_{6},(\{0.4\},\{0.3\},\{0.5\})\right)\right\}$ \\
$A_{3}$ & $\left\{\left(s_{7},(\{0.5\},\{0.3,0.4\},\{0.1\})\right)\right\}$ & $\left\{\left(s_{4},(\{0.2\},\{0.4\},\{0.3\})\right)\right\}$ \\
$A_{4}$ & $\left\{\left(s_{3},(\{0.5\},\{0.2\},\{0.2\})\right)\right\}$ & $\left\{\left(s_{6},(\{0.5\},\{0.2,0.3\},\{0.2\})\right)\right\}$ \\
$A_{5}$ & $\left\{\left(s_{2},(\{0.6\},\{0.4\},\{0.3\})\right)\right\}$ & $\left.\left\{\left(s_{4},(\{0.6\},\{0.3\},\{0.4\})\right)\right)\right\}$ \\
\hline & $C_{3}$ & $C_{4}$ \\
\hline$A_{1}$ & $\left\{\left(s_{3},(\{0.4\},\{0.4\},\{0.3\})\right)\right\}$ & $\left\{\left(s_{3},(\{0.5\},\{0.2\},\{0.4\})\right)\right\}$ \\
$A_{2}$ & $\left\{\left(s_{4},(\{0.3\},\{0.2\},\{0.4\})\right)\right\}$ & $\left\{\left(s_{5},(\{0.6\},\{0.1,0.2\},\{0.3\})\right)\right\}$ \\
$A_{3}$ & $\left\{\left(s_{3},(\{0.5\},\{0.3\},\{0.2\})\right)\right\}$ & $\left\{\left(s_{2},(\{0.3\},\{0.2\},\{0.3\})\right)\right\}$ \\
$A_{4}$ & $\left\{\left(s_{4},(\{0.4\},\{0.2\},\{0.1\})\right)\right\}$ & $\left\{\left(s_{3},(\{0.4\},\{0.1\},\{0.3\})\right),\left(s_{4},(\{0.3\},\{0.2\},\{0.2\})\right)\right\}$ \\
$A_{5}$ & $\left\{\left(s_{5},(\{0.7,0.8\},\{0.3\},\{0.3\})\right)\right\}$ &
\end{tabular}


Table 3. Decision matrix $D^{3}$.

\begin{tabular}{ccc}
\hline Alternative & $C_{1}$ & $C_{2}$ \\
\hline$A_{1}$ & $\left\{\left(s_{3},(\{0.3\},\{0.4\},\{0.5\})\right)\right\}$ & $\left\{\left(s_{3},(\{0.3\},\{0.4\},\{0.2\})\right)\right\}$ \\
$A_{2}$ & $\left\{\left(s_{5},(\{0.5\},\{0.2\},\{0.3\})\right)\right\}$ & $\left\{\left(s_{4},(\{0.7\},\{0.2\},\{0.2\})\right)\right\}$ \\
$A_{3}$ & $\left\{\left(s_{4},(\{0.4\},,\{0.1\},\{0.3\})\right),\left(s_{5},(\{0.6\},\{0.2\},\{0.1\})\right)\right\}$ & $\left\{\left(s_{5},(\{0.6\},\{0.1\},\{0.3\})\right)\right\}$ \\
$A_{4}$ & $\left\{\left(s_{6},(\{0.6\},\{0.1\},\{0.2\})\right)\right\}$ & $\left\{\left(s_{4},(\{0.5\},\{0.2\},\{0.2\})\right)\right\}$ \\
$A_{5}$ & $\left\{\left(s_{3},(\{0.4\},\{0.2\},\{0.3\})\right)\right\}$ & $\left\{\left(s_{6},(\{0.7,0.8\},\{0.2\},\{0.4\})\right)\right\}$ \\
\hline & $C_{3}$ & $C_{4}$ \\
\hline$A_{1}$ & $\left\{\left(s_{6},(\{0.2\},\{0.1,0.3\},\{0.6\})\right)\right\}$ & $\left\{\left(s_{5},(\{0.4\},\{0.3\},\{0.3\})\right)\right\}$ \\
$A_{2}$ & $\left\{\left(s_{2},(\{0.7\},\{0.2\},\{0.1\})\right)\right\}$ & $\left\{\left(s_{4},(\{0.5\},,\{0.2\},\{0.2\})\right),\left(s_{5},(\{0.6\},\{0.1\},\{0.2\})\right)\right\}$ \\
$A_{3}$ & $\left\{\left(s_{7},(\{0.6\},\{0.2\},\{0.2\})\right)\right\}$ & $\left\{\left(s_{2},(\{0.7\},\{0.1\},\{0.2\})\right)\right\}$ \\
$A_{4}$ & $\left\{\left(s_{4},(\{0.4,0.5\},\{0.1\},\{0.2\})\right)\right\}$ & $\left\{\left(s_{3},(\{0.6\},\{0.2,0.4\},\{0.3\})\right)\right\}$ \\
$A_{5}$ & $\left\{\left(s_{2},(\{0.6\},\{0.2\},\{0.4\})\right),\left(s_{3},(\{0.5\},\{0.2\},\{0.3\})\right)\right\}$ & $\left\{\left(s_{7},(\{0.8\},\{0.2\},\{0.1\})\right)\right\}$ \\
\hline
\end{tabular}

Table 4. Collective decision matrix $D$.

\begin{tabular}{|c|c|c|}
\hline Alternative & $C_{1}$ & $C_{2}$ \\
\hline$A_{1}$ & $\begin{array}{l}\left\{\left(s_{4.6635},(\{0.2661,0.2992\},\{0.3249\},\{0.3579\})\right),\right. \\
\left.\left(s_{5.0180},(\{0.3787,0.4068\},\{0.1988\},\{0.3100\})\right)\right\}\end{array}$ & $\left\{\left(s_{4.0090},(\{0.2992\},\{0.2870,0.3669\},\{0.2768\})\right)\right\}$ \\
\hline$A_{2}$ & $\left\{\left(s_{4.9455},(\{0.5012\},\{0.1271\},\{0.2656\})\right)\right\}$ & $\left\{\left(s_{5.009},(\{0.5819\},\{0.1876\},\{0.2768\})\right)\right\}$ \\
\hline$A_{3}$ & $\begin{array}{l}\left\{\left(s_{5.0635},(\{0.4109\},\{0.1817,0.2013\},\{0.2032\})\right),\right. \\
\left.\left(s_{5.4090},(\{0.4879\},\{0.2309,0.2557\},\{0.1390\})\right)\right\}\end{array}$ & $\begin{array}{l}\left\{\left(s_{4.0455},(\{0.4532\},\{0.2013\},\{0.3000\})\right),\right. \\
\left.\left(s_{4.3455},(\{0.3951\},\{0.2013\},\{0.3497\})\right)\right\}\end{array}$ \\
\hline$A_{4}$ & $\begin{array}{l}\left\{\left(s_{4.3365},(\{0.6029\},\{0.0000\},\{0.1625\})\right),\right. \\
\left.\left(s_{4.6365},(\{0.5671\},\{0.1574\},\{0.2000\})\right)\right\}\end{array}$ & $\left\{\left(s_{5.3090},(\{0.5324\},\{0.1625,0.1876\},\{0.2000\})\right)\right\}$ \\
\hline \multirow[t]{2}{*}{$A_{5}$} & $\left\{\left(s_{2.6455},(\{0.4340\},\{0.2888,0.3148\},\{0.2656\})\right)\right\}$ & $\left\{\left(s_{4.0910},(\{0.5716,0.6276\},\{0.2608,0.2843\},\{0.4277\})\right)\right\}$ \\
\hline & $C_{3}$ & $C_{4}$ \\
\hline$A_{1}$ & $\left\{\left(s_{3.7365},(\{0.2776,0.3059\},\{0.2273,0.3322\},\{0.4443\})\right)\right\}$ & $\begin{array}{l}\left\{\left(s_{4.291},(\{0.4657\},\{0.2301\},\{0.3622\})\right)\right. \\
\left.\left(s_{4.891},(\{0.4376\},\{0.2301\},\{0.3322\})\right)\right\}\end{array}$ \\
\hline$A_{2}$ & $\left\{\left(s_{3.0090},(\{0.5278\},\{0.2259\},\{0.2013\})\right)\right\}$ & $\left\{\left(s_{4.9545},(\{0.6037,0.6491\},\{0.1271,0.1625\},\{0.1876\})\right)\right\}$ \\
\hline$A_{3}$ & $\left\{\left(s_{4.9820},(\{0.5671\},\{0.1876\},\{0.2259\})\right)\right\}$ & $\left\{\left(s_{2.0000},(\{0.5013\},\{0.1574\},\{0.2608\})\right)\right\}$ \\
\hline$A_{4}$ & $\left\{\left(s_{3.4000},(\{0.5126,0.5424\},\{0.1574\},\{0.1271,0.1564\})\right)\right\}$ & $\begin{array}{l}\left\{\left(s_{3.0000},(\{0.5062\},\{0.1767,0.2245\},\{0.2656\})\right)\right\} \\
\left\{\left(s_{3.3545},(\{0.4785\},\{0.2259,0.2870\},\{0.2301\})\right)\right\}\end{array}$ \\
\hline$A_{5}$ & $\begin{array}{l}\left\{\left(s_{4.8635},(\{0.5921,0.6467\},\{0.2608\},\{0.3612\})\right),\right. \\
\left.\left(s_{5.2090}(\{0.5594,0.6184\},\{0.2608\},\{0.3270\})\right)\right\}\end{array}$ & $\begin{array}{l}\left\{\left(s_{5.7455},(\{0.6357\},\{0.2843\},\{0.2273\})\right)\right\} \\
\left\{\left(s_{6.0455},(\{0.5970\},\{0.2608\},\{0.2649\})\right)\right\}\end{array}$ \\
\hline
\end{tabular}

Table 5. Extended hesitant neutrosophic fuzzy linguistic decision matrix $\tilde{D}$.

\begin{tabular}{|c|c|c|}
\hline Alternative & $C_{1}$ & $C_{2}$ \\
\hline$A_{1}$ & $\begin{array}{l}\left\{\left(s_{4.6635},(\{0.2661,0.2992\},\{0.3249,0.3669\},\{0.3579,0.4443\})\right)\right) \\
\left.\left(s_{5} .0180,(\{0.3787,0.4068\},\{0.1988,0.3669\},\{0.3100,0.4443\})\right)\right\}\end{array}$ & $\begin{array}{l}\left\{\left(s_{4.0090},(\{0.2661,0.2992\},\{0.2870,0.3669\},\{0.2768,0.4443\})\right),\right. \\
\left.\left(s_{2} .0000,(\{0.2661,0.2661\},\{0.3669,0.3669\},\{0.4443,0.4443\})\right)\right\}\end{array}$ \\
\hline$A_{2}$ & $\left\{\left(s_{4.9455},(\{0.2661,0.5012\},\{0.1271,0.3669\},\{0.2656,0.4443\})\right)\right.$, & $\left\{\left(s_{5.009},(\{0.2661,0.5819\},\{0.1876,0.3669\},\{0.2768,0.4443\})\right)\right.$, \\
\hline & $\left.\left(s_{2.0000},(\{0.2661,0.2661\},\{0.3669,0.3669\},\{0.4443,0.4443\})\right)\right\}$ & $\left.\left(s_{2.0000},(\{0.2661,0.2661\},\{0.3669,0.3669\},\{0.4443,0.4443\})\right)\right\}$ \\
\hline$A_{3}$ & $\begin{array}{l}\left\{\left(s_{5.0635},(\{0.2661,0.4109\},\{0.1817,0.2013\},\{0.2032,0.4443\})\right),\right. \\
\left.\left.\left.\left(s_{5} .260 .2661,0.4879\right\},\{0.2309,0.2557\},\{0.1390,0.4443\}\right)\right)\right\}\end{array}$ & 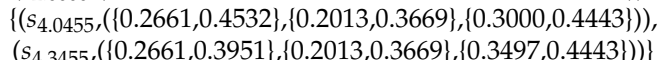 \\
\hline$A_{4}$ & $\left\{\left(s_{4.3365},(\{0.2661,0.6029\},\{0.0000,0.3669\},\{0.1625,0.4443\})\right)\right.$, & $\left\{\left(s_{5.3090},(\{0.2661,0.5324\},\{0.1625,0.1876\},\{0.2000,0.4443\})\right)\right.$, \\
\hline & $\left.\left(s_{4.6365},(\{0.2661,0.5671\},\{0.1574,0.3669\},\{0.2000,0.4443\})\right)\right\}$ & $\left.\left(s_{2.0000},(\{0.2661,0.2661\},\{0.3669,0.3669\},\{0.4443,0.4443\})\right)\right\}$ \\
\hline \multirow[t]{3}{*}{$A_{5}$} & $\left\{\left(s_{2.6455},(\{0.2661,0.4340\},\{0.2888,0.3148\},\{0.2656,0.4443\})\right)\right.$, & $\left\{\left(s_{4.0910},(\{0.5716,0.6276\},\{0.2608,0.2843\},\{0.4277,0.4443\})\right)\right.$, \\
\hline & $\left.\left(s_{2.0000},(\{0.2661,0.2661\},\{0.3669,0.3669\},\{0.4443,0.4443\})\right)\right\}$ & $\left.\left(s_{2.0000},(\{0.2661,0.2661\},\{0.3669,0.3669\},\{0.4443,0.4443\})\right)\right\}$ \\
\hline & $C_{3}$ & $C_{4}$ \\
\hline$A_{1}$ & $\begin{array}{l}\left\{\left(s_{3.7365},(\{0.2776,0.3059\},\{0.2273,0.3322\},\{0.4443,0.4443\})\right),\right. \\
\left\{\left(s_{2.0000},(\{0.2661,0.2661\},\{0.3669,0.3669\},\{0.4443,0.4443\})\right)\right\}\end{array}$ & $\begin{array}{l}\left\{\left(s_{4.291},(\{0.2661,0.4657\},\{0.2301,0.3669\},\{0.3622,0.4443\})\right),\right. \\
\left.\left(s_{4.891},(\{0.2661,0.4376),\{0.2301,0.3669\},\{0.3322,0.4443\})\right)\right\}\end{array}$ \\
\hline$A_{2}$ & $\begin{array}{l}\left\{\left(s_{3.0090}(\{0.2661,0.5278\},\{0.2259,0.3669\},\{0.2013,0.4443\})\right),\right. \\
\left.\left(s_{2} .0000,(\{0.2661,0.2661\},\{0.3669,0.3669\},\{0.4443,0.4443\})\right)\right\}\end{array}$ & $\begin{array}{l}\left\{\left(s_{4.9545},(\{0.6037,0.6491\},\{0.1271,0.1625\},\{0.1876,0.4443\})\right),\right. \\
\left.\left(s_{2} .0000,(\{0.2661,0.2661\},\{0.3669,0.3669\},\{0.4443,0.4443\})\right)\right\}\end{array}$ \\
\hline$A_{3}$ & $\left\{\left(s_{4.9820},(\{0.2661,0.5671\},\{0.1876,0.3669\},\{0.2259,0.4443\})\right)\right.$, & $\left\{\left(s_{2.0000},(\{0.2661,0.5013\},\{0.1574,0.3669\},\{0.2608,0.4443\})\right)\right.$, \\
\hline & $\left.\left(s_{2.0000},(\{0.2661,0.2661\},\{0.3669,0.3669\},\{0.4443,0.4443\})\right)\right\}$ & $\left.\left(s_{2.0000},(\{0.2661,0.2661\},\{0.3669,0.3669\},\{0.4443,0.4443\})\right)\right\}$ \\
\hline$A_{4}$ & $\left\{\left(s_{3.4000,},(\{0.5126,0.5424\},\{0.1574,0.3669\},\{0.1271,0.1564\})\right)\right.$, & $\left\{\left(s_{3.0000},(\{0.2661,0.5062\},\{0.1767,0.2245\},\{0.2656,0.4443\})\right)\right.$, \\
\hline$A_{5}$ & $\left\{\left(s_{4.8635},(\{0.5921,0.6467\},\{0.2608,0.3669\},\{0.3612,0.4443\})\right)\right.$, & $\left\{\left(s_{5.7455},(\{0.2661,0.6357\},\{0.2843,0.3669\},\{0.2273,0.4443\})\right)\right.$, \\
\hline & $5.2090,(\{0.5594,0.6184\},\{0.2608,0.3669\},\{0.3270,0.4443\}))\}$ & \\
\hline
\end{tabular}


Table 6. Decision results using different $\lambda$ with hesitant neutrosophic linguistic elements (HNLEs).

\begin{tabular}{|c|c|c|c|c|c|c|c|}
\hline$\lambda$ & & $A_{1}$ & $A_{2}$ & $A_{3}$ & $A_{4}$ & $A_{5}$ & Ranking \\
\hline \multirow{3}{*}{$\lambda=0.1$} & $d_{i}^{+}$ & 0.4762 & 0.4625 & 0.4409 & 0.3748 & 0.4462 & \multirow{3}{*}{$A_{4}>A_{5}>A_{3}>A_{1}>A_{2}$} \\
\hline & $d_{i}^{-}$ & 0.4386 & 0.4168 & 0.4605 & 0.4381 & 0.4786 & \\
\hline & $C C_{i}$ & 0.4795 & 0.4740 & 0.5109 & 0.5389 & 0.5175 & \\
\hline \multirow{3}{*}{$\lambda=0.5$} & $d_{i}^{+}$ & 0.4868 & 0.4793 & 0.4564 & 0.4355 & 0.4554 & \multirow{3}{*}{$A_{5}>A_{4}>A_{3}>A_{1}>A_{2}$} \\
\hline & $d_{i}^{-}$ & 0.4568 & 0.4452 & 0.4810 & 0.4720 & 0.4956 & \\
\hline & ${ }^{C} C_{i}$ & 0.4841 & 0.4816 & 0.5131 & 0.5201 & 0.5212 & \\
\hline \multirow{3}{*}{$\lambda=1$} & $d_{i}^{+}$ & 0.4999 & 0.4990 & 0.4749 & 0.4686 & 0.4670 & \multirow{3}{*}{$A_{5}>A_{4}>A_{3}>A_{1}>A_{2}$} \\
\hline & $d_{i}^{-}$ & 0.4772 & 0.4759 & 0.5043 & 0.5079 & 0.5144 & \\
\hline & ${ }^{C} C_{i}$ & 0.4884 & 0.4882 & 0.5150 & 0.5201 & 0.5242 & \\
\hline \multirow{3}{*}{$\lambda=2$} & $d_{i}^{+}$ & 0.5249 & 0.5344 & 0.5082 & 0.5190 & 0.4898 & \multirow{3}{*}{$A_{5}>A_{4}>A_{3}>A_{2}>A_{1}$} \\
\hline & $d_{i}^{-}$ & 0.5107 & 0.5231 & 0.5432 & 0.5624 & 0.5439 & \\
\hline & ${ }^{2} C_{i}$ & 0.4932 & 0.4947 & 0.5166 & 0.5200 & 0.5262 & \\
\hline \multirow{3}{*}{$\lambda=5$} & $d_{i}^{+}$ & 0.6142 & 0.6326 & 0.5922 & 0.6236 & 0.5561 & \multirow{3}{*}{$A_{5}>A_{4}>A_{3}>A_{2}>A_{1}$} \\
\hline & $d_{i}^{-}$ & 0.5725 & 0.6031 & 0.6171 & 0.6546 & 0.5930 & \\
\hline & ${ }^{C} C_{i}$ & 0.4824 & 0.4881 & 0.5103 & 0.5121 & 0.5160 & \\
\hline \multirow{3}{*}{$\lambda=10$} & $d_{i}^{+}$ & 0.6201 & 0.6616 & 0.5925 & 0.6746 & 0.5673 & \multirow{3}{*}{$A_{3}>A_{5}>A_{4}>A_{2}>A_{1}$} \\
\hline & $d_{i}^{-}$ & 0.6266 & 0.6714 & 0.6807 & 0.7290 & 0.6310 & \\
\hline & $C C_{i}$ & 0.5026 & 0.5037 & 0.5346 & 0.5194 & 0.5266 & \\
\hline
\end{tabular}

\subsection{Comparison Analysis}

Comparisons with other methods have been made [44,45]. If indeterminacy memberships are not considered, hesitant neutrosophic linguistic elements reduce to hesitant intuitionistic fuzzy linguistic elements (HIFLEs) [44] $h_{i j}^{\prime k}=\left\{\left(s_{\theta_{i j}^{k}},\left(I\left(s_{\theta_{i j}^{k}}\right), F\left(s_{\theta_{i j}^{k}}\right)\right)\right)\right\}$. We first aggregate hesitant intuitionistic fuzzy linguistic elements given by different DMs in different periods into collective ones $h_{i j}^{\prime}=\left\{\left(s_{\theta_{i j}}\right.\right.$, $\left.\left.\left(I\left(s_{\theta_{i j}}\right), F\left(s_{\theta_{i j}}\right)\right)\right)\right\}$ by using the generalized hesitant intuitionistic fuzzy linguistic correlated averaging (GHIFLCA) operator as in Equation (33).

$$
\begin{aligned}
& \operatorname{GHIFLCA}\left(h_{1}^{\prime}, h_{2}^{\prime}, \ldots, h_{n}^{\prime}\right)=\left(\sum_{j=1}^{n} m\left(A_{\sigma(j)}\right)-m\left(A_{\sigma(j-1)}\right)\left(h_{\sigma(j)}^{\prime}\right)^{\lambda}\right)^{1 / \lambda} \\
= & \bigcup_{a_{j}^{k} \in h_{j}}\left\{\left(s_{\left(\sum_{j=1}^{n}\left(m\left(A_{\sigma(j)}\right)-m\left(A_{\sigma(j-1)}\right)\right)\left(\theta_{\sigma(j)}^{k}\right)^{\lambda}\right)^{1 / \lambda}, \bigcup\left\{\left(\left(1-\prod_{j=1}^{n}\left(1-\left(\mu_{\sigma(j)}^{k}\right)^{\lambda}\right)^{m\left(A_{\sigma(j)}\right)-m\left(A_{\sigma(j-1)}\right)}\right)^{1 / \lambda},\right.\right.},\right.\right. \\
& \left.\left.\left.\left.1-\left(1-\prod_{j=1}^{n}\left(1-\left(1-\tau_{\sigma(j)}^{k}\right)^{\lambda}\right)^{m\left(A_{\sigma(j)}\right)-m\left(A_{\sigma(j-1)}\right)}\right)^{1 / \lambda}\right)\right\}\right)\right\} .
\end{aligned}
$$

Assuming DMs are risk-averse, we extend the hesitant intuitionistic fuzzy linguistic elements by adding the smallest linguistic evaluation values, the smallest truth-memberships, and the largest false-memberships. Determine the hesitant intuitionistic fuzzy linguistic positive ideal solution (HIFLPIS) $h^{\prime+}$ and the hesitant intuitionistic fuzzy linguistic negative ideal solution (HIFLNIS) $h^{\prime-}$.

HIFLPIS: $h^{\prime+}=\left\{\left\{\left(s_{9},(\{1,1\},\{0,0\})\right),\left(s_{9},(\{1,1\},\{0,0\})\right)\right\},\left\{\left(s_{9},(\{1,1\},\{0,0\})\right),\left(s_{9},(\{1,1\}\right.\right.\right.$, $\left.\{0,0\}))\},\left\{\left(s_{9},(\{1,1\},\{0,0\})\right),\left(s_{9},(\{1,1\},\{0,0\})\right)\right\},\left\{\left(s_{9},(\{1,1\},\{0,0\})\right),\left(s_{9},(\{1,1\},\{0,0\})\right)\right\}\right\}$.

HIFLNIS: $h^{--}=\left\{\left\{\left(s_{1},\left(\{0,0\},\{1,1\},\left(s_{1},(\{0,0\},\{1,1\})\right)\right\},\left\{\left(s_{1},\left(\{0,0\},\{1,1\},\left(s_{1},(\{0,0\},\{1\right.\right.\right.\right.\right.\right.\right.$, $1\}))\},\left\{\left(s_{1},\left(\{0,0\},\{1,1\},\left(s_{1},(\{0,0\},\{1,1\})\right)\right\},\left\{\left(s_{1},\left(\{0,0\},\{1,1\},\left(s_{1},(\{0,0\},\{1,1\})\right)\right\}\right\}\right.\right.\right.$.

Calculate the distances of the collective evaluation values to $h^{\prime+}$ and $h^{\prime-}$ by using Equations (34) and (35). The results are shown in Table 7.

$$
\begin{aligned}
d_{i}\left(h_{i}^{\prime}, h^{\prime+}\right)= & \left(\sum _ { j = 1 } ^ { n } ( m ( B _ { \sigma ( j ) } ) - m ( B _ { \sigma ( j - 1 ) } ) ) \frac { 1 } { 3 l } \sum _ { i = 1 } ^ { l } \left(\frac { 1 } { l _ { \theta } } \sum _ { t = 1 } ^ { l _ { \theta } } \left(\left|\theta_{\sigma(j i)}^{t}-9\right|^{\lambda} / 9^{\lambda}+\right.\right.\right. \\
& \left.\left.\left.\frac{1}{l_{t}} \sum_{k=1}^{l_{t}}\left|\mu_{\sigma(j i k)}^{t}-1\right|^{\lambda}+\frac{1}{l_{t}} \sum_{k=1}^{l_{t}}\left|\tau_{\sigma(j i k)}^{t}-0\right|^{\lambda}\right)\right)\right)^{1 / \lambda} .
\end{aligned}
$$




$$
\begin{aligned}
d_{i}\left(h_{i}^{\prime}, h^{\prime-}\right)= & \left(\sum _ { j = 1 } ^ { n } ( m ( B _ { \sigma ( j ) } ) - m ( B _ { \sigma ( j - 1 ) } ) ) \frac { 1 } { 3 l } \sum _ { i = 1 } ^ { l } \left(\frac { 1 } { l _ { \theta } } \sum _ { t = 1 } ^ { l _ { \theta } } \left(\left|\theta_{\sigma(j i)}^{t}-1\right|^{\lambda} / 9^{\lambda}+\right.\right.\right. \\
& \left.\left.\left.\frac{1}{l_{t}} \sum_{k=1}^{l_{t}}\left|\mu_{\sigma(j i k)}^{t}-0\right|^{\lambda}+\frac{1}{l_{t}} \sum_{k=1}^{l_{t}}\left|\tau_{\sigma(j i k)}^{t}-1\right|^{\lambda}\right)\right)\right)^{1 / \lambda} .
\end{aligned}
$$

Calculate the closeness coefficients of alternatives by using Equation (32). The results are also shown in Table 7. From the results, we can get different ranking results if indeterminacy memberships are not considered. In most cases, $A_{4}$ is the optimal alternative and $A_{5}$ becomes the suboptimal alternative. If $\lambda=10, A_{3}$ is the optimal alternative, and $A_{5}$ is ranked as the second to last.

Table 7. Decision results using different $\lambda$ with hesitant intuitionistic fuzzy linguistic elements (HIFLEs).

\begin{tabular}{cccccccc}
\hline \multicolumn{1}{c}{$\lambda$} & $\boldsymbol{C} C_{\boldsymbol{i}}$ & $\boldsymbol{A}_{\mathbf{1}}$ & $\boldsymbol{A}_{\mathbf{2}}$ & $\boldsymbol{A}_{\mathbf{3}}$ & $\boldsymbol{A}_{\mathbf{4}}$ & $\boldsymbol{A}_{\mathbf{5}}$ & Ranking \\
\hline$\lambda=0.1$ & $C C_{i}$ & 0.4110 & 0.4295 & 0.4556 & 0.4787 & 0.4678 & $A_{4}>A_{5}>A_{3}>A_{2}>A_{1}$ \\
$\lambda=0.5$ & $C C_{i}$ & 0.4158 & 0.4369 & 0.4589 & 0.4809 & 0.4718 & $A_{4}>A_{5}>A_{3}>A_{2}>A_{1}$ \\
$\lambda=1$ & $C C_{i}$ & 0.4212 & 0.4443 & 0.4626 & 0.4838 & 0.4756 & $A_{4}>A_{5}>A_{3}>A_{2}>A_{1}$ \\
$\lambda=2$ & $C C_{i}$ & 0.4300 & 0.4541 & 0.4686 & 0.4891 & 0.4800 & $A_{4}>A_{5}>A_{3}>A_{2}>A_{1}$ \\
$\lambda=5$ & $C C_{i}$ & 0.4444 & 0.4659 & 0.4791 & 0.4997 & 0.4796 & $A_{4}>A_{5}>A_{3}>A_{2}>A_{1}$ \\
$\lambda=10$ & $C C_{i}$ & 0.4775 & 0.4812 & 0.5026 & 0.5021 & 0.4705 & $A_{3}>A_{4}>A_{2}>A_{5}>A_{1}$ \\
\hline
\end{tabular}

If the linguistic arguments are not considered, the hesitant neutrosophic linguistic elements reduce to the hesitant neutrosophic elements [45] $h^{\prime \prime \prime}{ }_{i j}^{k}=\left\{\left(T_{i j}^{k} I_{i j}^{k}, F_{i j}^{k}\right)\right\}$. The hesitant neutrosophic elements are first aggregated into collective ones by using the generalized hesitant neutrosophic fuzzy correlated averaging (GHNFCA) operator as follows:

$$
\begin{gathered}
\operatorname{GHNFCA}\left(h_{1}^{\prime \prime}, h_{2}^{\prime \prime}, \ldots, h_{n}^{\prime \prime}\right)=\left(\sum_{j=1}^{n} m\left(A_{\sigma(j)}\right)-m\left(A_{\sigma(j-1)}\right)\left(h_{\sigma(j)}^{\prime \prime}\right)^{\lambda}\right)^{1 / \lambda} \\
=\bigcup_{a_{j}^{k} \in h_{j}}\left\{U \left\{\left(\left(1-\prod_{j=1}^{n}\left(1-\left(\mu_{\sigma(j)}^{k}\right)^{\lambda}\right)^{m\left(A_{\sigma(j)}\right)-m\left(A_{\sigma(j-1)}\right)}\right)^{1 / \lambda}, 1-\left(1-\prod_{j=1}^{n}(1-(1-\right.\right.\right.\right. \\
\left.\left.\left.\left.\left.\left.\left.v_{\sigma(j)}^{k}\right)^{\lambda}\right)^{m\left(A_{\sigma(j)}\right)-m\left(A_{\sigma(j-1)}\right)}\right)^{1 / \lambda}, 1-\left(1-\prod_{j=1}^{n}\left(1-\left(1-\tau_{\sigma(j)}^{k}\right)^{\lambda}\right)^{m\left(A_{\sigma(j)}\right)-m\left(A_{\sigma(j-1)}\right)}\right)^{1 / \lambda}\right)\right\}\right)\right\} .
\end{gathered}
$$

The DMs are also assumed to be risk-averse and the smallest truth-membership, the largest indeterminacy-membership, and the largest falsity-membership are added. Determine the hesitant neutrosophic fuzzy positive ideal solution $h^{\prime \prime}$ and hesitant neutrosophic fuzzy negative ideal solution $h^{\prime \prime}$. Calculate the distances of collective neutrosophic fuzzy values to $h^{\prime \prime+}$ and $h^{\prime \prime-}$ by the following equations:

$$
\begin{aligned}
d_{i}\left(h_{i}^{\prime \prime}, h^{\prime \prime+}\right)= & \left(\sum _ { j = 1 } ^ { n } ( m ( B _ { \sigma ( j ) } ) - m ( B _ { \sigma ( j - 1 ) } ) ) \frac { 1 } { 3 l } \sum _ { i = 1 } ^ { l } \left(\frac { 1 } { l _ { \theta } } \sum _ { t = 1 } ^ { l _ { \theta } } \left(\left|\mu_{\sigma(j i)}^{t}-1\right|^{\lambda}+\right.\right.\right. \\
& \left.\left.\left.\frac{1}{l_{t}} \sum_{k=1}^{l_{t}}\left|v_{\sigma(j i k)}^{t}-0\right|^{\lambda}+\frac{1}{l_{t}} \sum_{k=1}^{l_{t}}\left|\tau_{\sigma(j i k)}^{t}-0\right|^{\lambda}\right)\right)\right)^{1 / \lambda} . \\
d_{i}\left(h_{i}^{\prime \prime}, h^{\prime \prime-}\right)= & \left(\sum _ { j = 1 } ^ { n } ( m ( B _ { \sigma ( j ) } ) - m ( B _ { \sigma ( j - 1 ) } ) ) \frac { 1 } { 3 l } \sum _ { i = 1 } ^ { l } \left(\frac { 1 } { l _ { \theta } } \sum _ { t = 1 } ^ { l _ { \theta } } \left(\left|\mu_{\sigma(j i)}^{t}-0\right|^{\lambda}+\right.\right.\right. \\
& \left.\left.\left.\frac{1}{l_{t}} \sum_{k=1}^{l_{t}}\left|v_{\sigma(j i k)}^{t}-1\right|^{\lambda}+\frac{1}{l_{t}} \sum_{k=1}^{l_{t}}\left|\tau_{\sigma(j i k)}^{t}-1\right|^{\lambda}\right)\right)\right)^{1 / \lambda} .
\end{aligned}
$$

Other steps are the same as the proposed methods. If $\lambda=2$, we can get the results as $C C_{1}=0.7661$, $C C_{1}=0.7229, C C_{1}=0.7686, C C_{1}=0.6880$, and $C C_{1}=0.7256$. The alternatives can be ranked as $A_{3}>A_{1}>A_{5}>A_{2}>A_{4}$.

Results of different methods are listed in Table 8 if $\lambda=2$. From the results, we can see that different ranking results can be obtained using different methods. In the method of [45], only hesitant neutrosophic fuzzy values have been considered. In the method of [44], the memberships are in the form of intuitionistic fuzzy values. In fact, DMs would like to evaluate with linguistic arguments in the evaluation process. By using hesitant neutrosophic fuzzy elements to model fuzziness and uncertainty, more accuracy results can be obtained. Decision results are more reasonable in the proposed method. 
Based on comparison with existing methods, therefore, this paper presents a new way of solving multiple attribute decision-making in a hesitant neutrosophic linguistic environment.

Table 8. Results of different methods.

\begin{tabular}{cc}
\hline Method & Ranking \\
\hline Method from [44] & $A_{4}>A_{5}>A_{3}>A_{2}>A_{1}$ \\
Method from [45] & $A_{3}>A_{1}>A_{5}>A_{2}>A_{4}$ \\
Proposed method & $A_{5}>A_{4}>A_{3}>A_{2}>A_{1}$ \\
\hline
\end{tabular}

\section{Conclusions}

In this paper, we first introduce a definition of the hesitant neutrosophic fuzzy linguistic set. We then define some distance measures including the hesitant neutrosophic linguistic Euclidean distance, the hesitant neutrosophic linguistic Hamming distance, and the generalized hesitant neutrosophic linguistic distance. We also define some correlated distance measures including the generalized hesitant neutrosophic linguistic correlation averaging distance and the generalized hesitant neutrosophic linguistic hybrid correlation averaging distance. We present some correlated aggregation operators for hesitant neutrosophic fuzzy linguistic set including the HNLCA operator, the HNLCGA operator, the GHNLCA operator, and the QHNLCA operator. A new hesitant neutrosophic fuzzy linguistic multiple attribute decision-making method has been developed based on the new distance measures and the new aggregation operators. We apply the new method to solve investment problems. Two special cases of the hesitant neutrosophic fuzzy linguistic set from existing works have been used in the numerical example to illustrate the difference between the proposed method and several other methods.

The proposed method has the following advantages. First, the hesitant neutrosophic fuzzy linguistic set is the extension of the hesitant fuzzy set, the neutrosophic set, and the linguistic term set, which can be applied in decision problems with imprecise, uncertain, incomplete, and inconsistent information. Compared with other tools, the hesitant neutrosophic fuzzy linguistic set is more flexible and accurate, and many existing tools are special cases of the hesitant neutrosophic fuzzy linguistic set. Second, correlation exists extensively in the decision-making process, which can be modeled by using the Choquet integral. We combine the Choquet integral with the hesitant neutrosophic fuzzy linguistic theory to develop some distance measures and aggregation operators. Finally, the new multiple attribute group decision-making method is based on the TOPSIS and the Choquet integral for hesitant neutrosophic fuzzy linguistic information, which can be used to solve complicated decision problems.

Acknowledgments: This work was partly supported by National Natural Science Foundation of China (No. 11401757), the Postdoctoral Science Foundation of China (2015M582624), and the Shaanxi Province Postdoctoral Science Foundation.

Author Contributions: Yongfeng Pang conceived the idea; Wei Yang wrote the paper and revised the paper. The authors have read and approved the final manuscript.

Conflicts of Interest: The authors declare no conflict of interest.

\section{References}

1. Smarandache, F. A unifying field in logics. In Neutrosophy: Neutrosophic Probability, Set and Logic; American Research Press: Rehoboth, DE, USA, 1998.

2. Atanassov, K.; Gargov, G. Interval-valued intuitionistic fuzzy sets. Fuzzy Sets Syst. 1989, 31, 343-349.

3. Liu, P.D.; Tang, G.L. Some power generalized aggregation operators based on the interval neutrosophic sets and their application to decision making. J. Intell. Fuzzy Syst. 2016, 30, 2517-2528.

4. Biswas, P.; Pramanik, S.; Giri, B.C. TOPSIS method for multi-attribute group decision-making under single-valued neutrosophic environment. Neural Comput. Appl. 2016, 27, 727-737. 
5. Yang, H.L.; Guo, Z.L.; She, Y.H.; Liao, X.W. On single valued neutrosophic relations. J. Intell. Fuzzy Syst. 2016, 30, 1045-1056.

6. Thamaraiselvi, A.; Santhi, R. A New Approach for Optimization of Real Life Transportation Problem in Neutrosophic Environment. Math Probl. Eng. 2016, 2016, 5950747, doi:10.1155/2016/5950747.

7. Zhao, A.W.; Du, J.G.; Guan, H.J. Interval valued neutrosophic sets and multi-attribute decision-making based on generalized weighted aggregation operator. J. Intell. Fuzzy Syst. 2015, 29, 2697-2706.

8. Yang, W.; Shi, J.R.; Pang, Y.F.; Zheng, X. Linear assignment method for interval neutrosophic sets. Neural Comput. Appl. 2016, 1-12, doi:10.1007/s00521-016-2575-2.

9. Ye, J. An extended TOPSIS method for multiple attribute group decision making based on single valued neutrosophic linguistic numbers. J. Intell. Fuzzy Syst. 2015, 28, 247-255.

10. Shi, L.L.; Ye, J. Cosine Measures of Linguistic Neutrosophic Numbers and Their Application in Multiple Attribute Group Decision-Making. Information 2017, 8, 117, doi:10.3390/info8040117.

11. Ye, J. Trapezoidal neutrosophic set and its application to multiple attribute decision-making. Neural Comput. Appl. 2015, 26, 1157-1166.

12. Guo, Y.H.; Sengür, A. A novel image segmentation algorithm based on neutrosophic similarity clustering. Appl. Soft Comput. 2014, 25, 391-398.

13. Karaaslan, F. Correlation coefficients of single-valued neutrosophic refined soft sets and their applications in clustering analysis. Neural Comput. Appl. 2017, 28, 2781-2793.

14. Sun, H.C.; Sun, M. Simplified neutrosophic weighted Maclaurin symmetric mean and its application to supply chain management. ICIC Express Lett. 2015, 9, 3221-3227.

15. Liu, P.D.; Shi, L.L. The generalized hybrid weighted average operator based on interval neutrosophic hesitant set and its application to multiple attribute decision making. Neural Comput. Appl. 2015, 26, 457-471.

16. Liu, P.D.; Chu, Y.C.; Li, Y.W.; Chen, Y.B. Some Generalized Neutrosophic Number Hamacher Aggregation Operators and Their Application to Group Decision Making. Int. J. Fuzzy Syst. 2014, 16, 242-255.

17. Sun, H.X.; Yang, H.X.; Wu, J.Z.; Yao, O.Y. Interval neutrosophic numbers Choquet integral operator for multi-criteria decision making. J. Intell. Fuzzy Syst. 2015, 28, 2443-2455.

18. Peng, J.J.; Wang, J.Q.; Wu, X.H.; Wang, J.; Chen, X.H. Multi-valued Neutrosophic Sets and Power Aggregation Operators with Their Applications in Multi-criteria Group Decision-making Problems. Int. J. Comput. Syst. 2015, 8, 345-363.

19. Ye, J. Similarity measures between interval neutrosophic sets and their applications in multicriteria decision-making. J. Intell. Fuzzy Syst. 2014, 26, 165-172.

20. Ye, J. Single-valued neutrosophic cross-entropy for multicriteria decision making problems. Appl. Math. Model. 2014, 38, 1170-1175.

21. Ye, J. Multicriteria decision-making method using the correlation coefficient under single-valued neutrosophic environment. Int. J. Gen. Syst. 2013, 42, 386-394.

22. Zhang, H.Y.; Ji, P.; Wang, J.Q.; Chen, X.H. An Improved Weighted Correlation Coefficient Based on Integrated Weight for Interval Neutrosophic Sets and its Application in Multicriteria Decision-making Problems. Int. J. Comput. Syst. 2013, 8, 1027-1043.

23. Şahin, R. Cross-entropy measure on interval neutrosophic sets and its applications in multicriteria decision making. Neural Comput. Appl. 2017, 28, 2781-2793.

24. Majumdar, P.; Samanta, S.K. On similarity and entropy of neutrosophic sets. J. Intell. Fuzzy Syst. 2014, 26, 1245-1252.

25. Şahin, R.; Kücük, A. On similarity and entropy of neutrosophic soft sets. J. Intell. Fuzzy Syst. 2014, 27, 2417-2430.

26. Tian, Z.P.; Zhang, H.Y.; Wang, J.; Wang, J.Q.; Chen, X.H. Multi-criteria decision-making method based on a cross-entropy with interval neutrosophic sets. Int. J. Syst. Sci. 2016, 47, 3598-3608.

27. Torra, V. Hesitant fuzzy sets. Int. J. Intell. Syst. 2010, 25, 529-539.

28. Torra, V.; Narukawa, Y. On hesitant fuzzy sets and decision. In Proceedings of the 18th IEEE International Conference on Fuzzy Systems, Jeju Island, Korea, 20-24 August 2009; pp. 1378-1382.

29. Farhadinia, B. Information measures for hesitant fuzzy sets and interval-valued hesitant fuzzy sets. Inf. Sci. 2013, 240, 129-144.

30. Tan, C.Q.; Yi, W.T.; Chen, X.H. Hesitant fuzzy Hamacher aggregation operators for multicriteriadecision making. Appl. Soft Comput. 2015, 26, 325-349. 
31. Wei, G.W. Hesitant fuzzy prioritized operators and their application to multiple attribute decision making. Knowl. Based Syst. 2012, 31, 176-182.

32. Xu, Z.S.; Xia, M.M. On Distance and Correlation Measures of Hesitant Fuzzy Information. Int. J. Intell. Syst. 2011, 26, 410-425.

33. Xu, Z.S.; Zhang, X.L. Hesitant fuzzy multi-attribute decision making based on TOPSIS with incomplete weight information. Knowl. Based Syst. 2013, 52, 53-64.

34. Liu, P.D.; Mahmood, T.; Khan, Q. Multi-Attribute Decision-Making Based on Prioritized Aggregation Operator under Hesitant Intuitionistic Fuzzy Linguistic Environment. Symmetry 2017, 9, 270.

35. Herrera F.; Martínez, L. An approach for combining linguistic and numerical information based on 2-tuple fuzzy linguistic representation model in decision-making. Int. J. Uncertain. Fuzziness Knowl. Based Syst. 2000, 8, 539-562.

36. $\mathrm{Xu}, \mathrm{Z}$.S. Uncertain linguistic aggregation operators based approach to multiple attribute group decision making under uncertain linguistic environment. Inf. Sci. 2004, 168, 171-184.

37. Rodríguez, R.M.; Martínez, L.; Herrera, F. Hesitant Fuzzy Linguistic Term Sets for Decision Making. IEEE Trans. Fuzzy Syst. 2012, 20, 109-119.

38. Delgado, M.; Verdegay, J.L.; Vila, M.A. On aggregation operations of linguistic labels. Int. J. Intell. Syst. 1993, 8, 351-370.

39. Yang, W.; Shi, J.R.; Pang, Y.F. Generalized Linguistic Hesitant Intuitionistic Fuzzy Hybrid Aggregation Operators. Math. Probl. Eng. 2015, 2015, 983628, doi:10.1155/2015/983628.

40. Ye, J. Hesitant interval neutrosophic linguistic set and its application in multiple attribute decision making. Int. J. Mach. Learn. Cybern. 2017, 1-12, doi:10.1007/s13042-017-0747-8.

41. Wang, Z.Y.; Klir, G. Fuzzy Measure Theory; Plenum Press: New York, NY, USA, 1992.

42. Sugeno, M. Theory of Fuzzy Integrals and Applications; Tokyo Institute of Technology: Tokyo, Japan, 1974.

43. Yang, W. Induced quasi-arithmetic uncertain linguistic aggregation operator. Int. J. Uncertain. Fuzziness Knowl. Based Syst. 2013, 21, 55-77.

44. Yang, W.; Pang, Y.F.; Shi, J.R.; Wang, C.J. Linguistic hesitant intuitionistic fuzzy decision-making method based on VIKOR. Neural Comput. Appl. 2018, 29, 613-626

45. Ye, J. Multi-attribute decision making method under a single-valued neutrosophic hesitant fuzzy environment. J. Intell. Syst. 2015, 24, 23-34.

(C) 2018 by the authors. Licensee MDPI, Basel, Switzerland. This article is an open access article distributed under the terms and conditions of the Creative Commons Attribution (CC BY) license (http://creativecommons.org/licenses/by/4.0/). 